\title{
The X-ray plateau phase of gamma-ray burst originating from an expanding shell with a Lorentz factor of a few tens
}

Hüsne Dereli-Bégué ( $\square$ husnedereli@gmail.com )

Bar-llan University https://orcid.org/0000-0002-8852-7530

\section{Asaf Pe'er}

Bar-llan University

\section{Felix Ryde}

KTH Royal Institute of Technology

\section{Sam R. Oates}

University of Birmingham

\section{Bing Zhang}

University of Nevada, Las Vegas https://orcid.org/0000-0002-9725-2524

\section{Maria Dainotti}

National Astronomical Observatory of Japan

\section{Article}

Keywords: Gamma-ray bursts, Lorentz factor, X-ray, optical bands

Posted Date: November 17th, 2021

DOI: https://doi.org/10.21203/rs.3.rs-993344/v1

License: (a) This work is licensed under a Creative Commons Attribution 4.0 International License. Read Full License

Version of Record: A version of this preprint was published at Nature Communications on September 24th, 2022. See the published version at https://doi.org/10.1038/s41467-022-32881-1. 


\title{
The X-ray plateau phase of gamma-ray burst originating from an expanding shell with a Lorentz factor of a few tens
}

\author{
Hüsne Dereli-Bégué ${ }^{1,{ }^{*}}$, Asaf Pe'er ${ }^{1}$, Felix Ryde ${ }^{2}$, Samantha R. Oates ${ }^{3}$, Bing Zhang ${ }^{4}$, and \\ Maria G. Dainotti ${ }^{5}$
}

\author{
${ }^{1}$ Department of Physics, Bar-Ilan University, Ramat-Gan 52900, Israel \\ ${ }^{2}$ Department of Physics, KTH Royal Institute of Technology and The Oskar Klein Centre, SE-106 91 Stockholm, \\ Sweden \\ ${ }^{3}$ School of Physics and Astronomy \& Institute for Gravitational Wave Astronomy, University of Birmingham, \\ Birmingham B15 2TT, UK \\ ${ }^{4}$ Department of Physics and Astronomy, University of Nevada, Las Vegas, NV 89154, USA \\ ${ }^{5}$ National Astronomical Observatory of Japan, 2-21-1 Osawa, Mitaka, Tokyo 181-8588 \\ *husnedereli@gmail.com
}

\section{ABSTRACT}

Gamma-ray bursts (GRBs) are one of the most energetic explosions known in the Universe and are also known to have the most relativistic jets, with initial expansion Lorentz factors of $100<\Gamma_{i}<1000^{1-7}$. Many of these objects have a plateau in their early X-ray light curves (up to thousands of seconds) ${ }^{8-12}$. In this phase, the $\mathrm{X}$-ray flux decreases much slower than theoretically expected ${ }^{13}$ which has puzzled the community for many years. Here, we show that the observed signal during this phase in both the X-ray and the optical bands is naturally obtained within the classical GRB "fireball" model, provided that (i) the initial Lorentz factor of the relativistically expanding jet is of the order of a few tens, rather than a few hundreds, as is often cited in the literature, and (ii) the expansion occurs into a medium-low density "wind" with density typically 3-4 orders of magnitude below the expectation from a Wolf-Rayet star ${ }^{14}$. Within this framework, the end of the "plateau" phase (the beginning of the regular afterglow) marks the transition from the coasting phase to the self-similar expansion phase, which follows the scaling laws first derived by Blandford \& McKee. ${ }^{15}$. This result therefore implies that the long GRB progenitors are either (i) not Wolf-Rayet stars, or (ii) the properties of the wind ejected by these stars prior to their final explosion are very different than the properties of the wind ejected at earlier times. This result shows that the range of Lorentz factors in GRB jets is much wider than previously thought, and bridges an observational 'gap' between mildly relativistic jets ${ }^{16}$ inferred in active galactic nuclei, $\Gamma_{i} \lesssim 20$, to the much higher Lorentz factors, $\Gamma_{i} \lesssim 1000$ inferred in a few extreme GRBs ${ }^{7}$.

\section{MAIN TEXT}

One of the most puzzling results in the study of gamma-ray bursts (GRBs) is the existence of a long "plateau" in the early X-ray light curve (up to thousands of seconds) of a significant fraction $43 \%$ of GRBs until $2009^{17}$ and 56\% until $2019^{12}$. This plateau, not predicted theoretically, was first detected by the Neil Gehrels Swift Observatory ${ }^{18}$ in 2005, and despite the long time passed since its discovery, its origin is still highly debate in the literature, with many authors suggesting various extensions to the classical "fireball" model in order to explain it.

To investigate this issue, we use the sample of 222 GRBs with known redshifts and plateau phases as defined in Ref. ${ }^{12}$. These GRBs were detected by the Neil Gehrels Swift Observatory from January 2005 
until August 2019. They represent 56\% of all GRBs with known redshifts observed by the Swift satellite in this period.

In order to make our analysis as reliable as possible, we limit the bursts used to the ones having the best quality observations. Therefore, we added three criteria to the one used by Ref. ${ }^{12}$. We require (i) a long lasting (from $10^{2}$ to $10^{5} \mathrm{~s}$ ) plateau phase with a temporal X-ray slope larger than -0.7 , followed by a power-law decay phase at later times (the self-similar phase). (ii) Sufficient number of data points ( $\gtrsim 5)$ during the plateau and self-similar phases to enable the fits to give well constrained parameter values (see "Methods" below). For this to be valid, we exclude all X-ray flares from the analyzed data. We found 130 GRBs matching these criteria. (iii) Finally, we require an optical counterpart at around the same time as the X-ray data. We searched the optical catalogue of Ref. ${ }^{19}$ and found 24 GRBs in our sample to have optical counterpart. Out of these, we had full access to the optical data of 13 GRBs, which are listed in Tables 1, 2, 3 .

The key to understanding the observations is the realization that the end of the plateau corresponds to the transition from a coasting phase (steady state in which all the energy has been converted to kinetic energy) to a self-similar expansion phase (decaying phase in which the kinetic energy converted back to the radiation by the shocks) of the expanding plasma. The observations originate entirely from ambient electrons collected and heated by the forward shock wave, propagating at relativistic speeds inside a "wind" (decaying density) ambient medium. As we show (see Method - 'Theory' below,) this assumption about the decay of the ambient density is crucial in explaining the observations. During the transition from the coasting to the decaying phases a reverse shock crosses the expanding plasma. However, the contribution from electrons heated by the reverse shock is suppressed due to (i) the declining ambient density, which implies that the ratio of plasma density to ambient density remains constant (in a conical expansion), and (ii) its slower speed, which translates into less energetic electrons that emit at much lower frequencies than forward shock heated electrons, implying that the contribution to the optical and X-ray bands is negligible.

In analyzing the optical and X-ray light curves, we therefore expect two achromatic temporal breaks: one during the transition from the coasting to the self-similar expansion (this marks the end of the "plateau"); and a second, later break is the expected jet-break ${ }^{10}$. The temporal slopes during the plateau phase, self-similar phase and after the jet break are marked as $\alpha_{\mathrm{p}}, \alpha_{\mathrm{A} 1}$ and $\alpha_{\mathrm{A} 2}$ respectively and presented in Tables $1-3$. The transition times are marked as $T_{\mathrm{a}}$ and $T_{\mathrm{b}}$ (in some bursts a second break could not be detected due to poor data quality at very late times).

The optical and X-ray light curves do not necessarily follow the same power law in either of the dynamical phases (plateau or late-time afterglow). This can easily be understood in the framework of synchrotron emission from forward-shock accelerated electrons. The electrons assume a power law distribution with power law index $p$, namely $N_{e l}(\gamma) d \gamma \propto \gamma^{-p}$ above a minimum value $\gamma_{m}$; below this value, it is safe to assume that the electrons have a Maxwellian (or quasi-Maxwellian) energy distribution ${ }^{13,20}$. This assumption leads to a broken power law spectra and light curves, whose shapes, in the relevant observed bands, depend on whether the peak frequency, $v_{m}$ (corresponding to $\gamma_{m}$ ) is above or below the cooling frequency, $v_{c}$ (corresponding to cooling $\gamma_{c}$ for which the rate of energy lost by synchrotron emission is equal to the rate of energy lost by adiabatic cooling). For a given observed frequency (optical [typically, U-band]: $v_{\mathrm{U}}$ or X-rays, $v_{\mathrm{X}}$ ), different possibilities of the expected light curve and spectra exist, and are summarized in Table 4 . The fast cooling regimes $\left(v_{m}>v_{c}\right)$ are marked as $[\mathrm{A}],[\mathrm{B}],[\mathrm{C}]$ and the slow cooling regimes $\left(v_{m}<v_{c}\right)$ are marked as [D], [E], [F], respectively. At low frequencies, one needs to consider synchrotron self-absorption, which can safely be neglected being below the optical frequency at all observed times (see details in Methods, 'Theoretical model').

When analyzing the data, we split the sample into 3 categories based on the X-ray and optical light 
curves during the plateau phase (corresponding to Tables $1-3$ ). These categories match very well the theoretical predictions. Case I: a flat X-ray light curve $\left(F_{V} \propto t_{\text {obs. }}^{0.0 .-0.2}\right)$, corresponding to regions [C] and $[\mathrm{F}]$ in Table 4 and decaying optical light curve $\left(F_{V} \propto t_{\text {obs. }}^{-0.5-0.7}\right)$, region [E] in Table 4. Theoretically, this case corresponds to cooling frequency in between the optical and the X-ray bands, namely $v_{\mathrm{U}}<v_{c}<v_{\mathrm{X}}$. Case II: both the X-ray and the optical light curves are flat. Theoretically, this is expected for low cooling frequency, $v_{c}<v_{\mathrm{U}}<v_{\mathrm{X}}$. Case III: both X-ray and optical light curves are decaying. This is expected when the cooling frequency is high, $v_{\mathrm{U}}<v_{\mathrm{X}}<v_{c}$. Very interestingly, after excluding faint flares, all GRBs in our sample fall into one of these three categories. Furthermore, these categories, selected by the $\mathrm{X}$-ray and optical light curves, seem to be associated with a given burst energy and duration of the plateau; see Figure 1.

For each GRB, X-ray spectral slopes ( $\beta_{\mathrm{p}}$ for plateau phase, $\beta_{\mathrm{A} 1}$ for self-similar phase, $\beta_{\mathrm{A} 2}$ for the spectral slope after the jet break $^{21}$ ) for the same time range as the temporal slopes were obtained from the online Swift repository and optical spectral slopes were retrieved from the literature (references are given in Table $1-3)$. Using these spectral slopes, we further examine the validity of the theory by studying the temporal and spectral slopes in both the X-ray and optical bands, in both the plateau and the self-similar phases along side the theoretical predictions (the "closure relations") for the different relevant regimes ([C], $[\mathrm{E}],[\mathrm{F}])$ in Figures 2 and 3. From the Figures, it is clear that all the data - spectral and temporal, both in X-ray and optical are consistent with the theoretical closure relations of the model.

Furthermore from the closure relation, we deduced the power law index $p$ of the accelerated electrons. We did this by fitting the light curves of both the optical and X-ray during both the plateau and afterglow phases, under the assumption that the power law index of the accelerated electrons does not change in between these dynamical phases. We calculated the best fitted value, consistent with all 4 independent measurements, for both regions $[\mathrm{E}]$ and $[\mathrm{F}]$. This is demonstrated in Figures 4 and 5. We find that for all GRBs in our sample, the power law index, $p$ is in the range $1.8 \lesssim p \lesssim 2.5$. For those bursts whose X-ray light curve is identified as being in region [E], the electron power law index is narrowly clustered around $p \simeq 2$, while a larger spread is found for other GRBs. The results are summarized in Table 5 .

We find that the optical data of 5/13 GRBs in our sample (listed in Table 2) are compatible with being in region $[\mathrm{F}]$, implying that the cooling frequency at the end of the plateau phase is below the observed optical band, $v_{c}<v_{\mathrm{U}}$. For another 4/13 GRB (listed in Table 1), the optical light curve decays (corresponding region $[\mathrm{E}]$ ), while the $\mathrm{X}$-ray light curve is flat, namely for these bursts $v_{\mathrm{U}}<v_{c}<v_{\mathrm{X}}$. For the remaining 4/13 GRBs (listed Table 3) both the optical and X-ray light curves decay, and are therefore compatible with the cooling frequency being above the observed $\mathrm{X}$-ray band $v_{\mathrm{X}}<v_{c}$.

We use here a simple theoretical model: the well-studied synchrotron emission from a power law distribution of electrons accelerated at the forward shock, generated when the outflow propagates into a "wind" density profile, characterized by a decaying density, $\rho(r) \propto r^{-2}$. The excellent agreement between the data and the theory enables us to use the data to determine or constrain the parameters of the outflow and the wind, in particular the proportionality constant $A_{\star}$ of the wind density, the initial jet Lorentz factor, $\Gamma_{i}$, the fraction of energy in the electrons, $\varepsilon_{e}$ and the magnetization, $\varepsilon_{B}$. All relevant parameters used in the fits are given in Table 6 (see details in Methods 'Flux Ratio'). For the 9 GRBs in Tables 1 and 2, the $\mathrm{X}$-ray flux and the transition time that marks the end of the plateau phase, enable a direct deduction of $\varepsilon_{e}$, while for the 4 GRBs in Table 3, only a lower limit is available. For the 4 GRBs in Table 1 we can directly infer the combined value of $A_{\star} \Gamma_{i}^{4}$. For a given value of $A_{\star}$, the value of $\varepsilon_{B}$ is solely determined. Thus, an independent measure of $\varepsilon_{B}$ enables to break the degeneracy. In Figure 6 we use known limits $\left(0.001 \lesssim \varepsilon_{B} \leq 1.0\right)$ to constrain the values of $A_{\star}$ and $\Gamma_{i}$. For the 5 GRBs in Table 2, only a lower limit on the value of $\varepsilon_{B}$ can be deduced. This is still highly valuable, as physically $\varepsilon_{B}<1$. For the 4 GRBs in Table 3 , only an upper limit on $\varepsilon_{B}$ is obtained. We use these limits to constrain the combination of $A_{\star} \Gamma_{i}^{4}$. 
They are presented in Table 7 and Figure 6 . In the figure, the values of $\Gamma_{i}$ and $A_{\star}$ are marked by lines, each correspond to a different GRB. These are, from top to bottom: GRBs 080607, 110213A, 060714, 080310, 061121, 060605, 130831A, 091029, 050319, 060729, 060614, 100418A and 171205A. While directly deduced values are marked by squares, upper and lower values are marked by arrows.

A histogram of the $\Gamma_{i}$ for the 13 GRBs in our sample is shown in Figure 7 . The average value of the Lorentz factor deduced is $\left\langle\Gamma_{i}\right\rangle \approx 85$ (median is 68), although the range span is between $5 \leq \Gamma_{i} \leq 322$. These values may initially seem at odds with the typical values discussed in the literature, of $\Gamma_{i} \gtrsim 100$ of GRB jets. However, a closer look reveals that in fact there is no contradiction.

There are several ways of inferring the value of the Lorentz factor in GRB jets ${ }^{22}$. The most widely used method is the opacity argument ${ }^{1-6}$, which is commonly used in deducing that observed GeV (LAT) photons must originate from a region expanding at $\Gamma \gtrsim 100^{\prime} s$. This argument, though, is only valid when $>\mathrm{MeV}$ photons are observed. A previous search ${ }^{23}$ of GRBs observed from 2008 until May 2016 by Fermi-LAT that appeared in the $2^{\text {nd }}$ catalogue ${ }^{24}$ which are fitted with a broken power behaviour, have the Test Statistic $T S>64$ and known redshift (in total 13 GRBs) demonstrates that 3 GRBs, out of the 13 show evidence for a shallow decay phase in the LAT data; only one (the hard-short GRB 090510) show any evidence for a decaying plateau in the Swift-XRT data. For this specific burst, the X-ray slope during the early afterglow can only marginally identify it as having a plateau, (X-ray slope of $0.69_{-0.06}^{+0.05}$, vs. the -0.7 limit used in this study). These are consistent with earlier findings by Ref. ${ }^{25}$ for 26 GRBs triggered by Swift-BAT and subsequently detected by Fermi-LAT ${ }^{24}$ and/or Imaging Atmospheric Cherenkov Telescopes.

The second method relies on identifying an early optical flash as originating from the reverse shock $^{7,26-29}$. Since the reverse shock exists during the transition from the coasting to the decaying (self-similar) phase, identifying emission from it indicates the transition time, from which, assuming the energy and ambient density are known, the initial Lorentz factor can be deduced. However, a clear signature of a reverse shock emission is nearly never identified ${ }^{30-32}$ (as opposed to flares common to both the X-ray and optical data ${ }^{33-37}$ and does not exist in any of the bursts in our sample.

When a strong thermal component exists during the prompt phase, it is possible to use it to infer the Lorentz factor at the initial phase of the expansion ${ }^{38}$. We therefore searched (i) all bursts with known strong thermal component as appeared in Refs. ${ }^{39-41}$. None of those showed any evidence for an X-ray plateau. (ii) Similarly, none of the bursts in our sample show any evidence for a thermal emission.

To conclude, we find that in all cases where there is any evidence for an initial Lorentz factor $\Gamma_{i} \gtrsim a$ few hundreds, no X-ray plateau exists, and vice versa: for all bursts that show a plateau, no significant indication for high Lorentz factor exist, neither at high energy, thermal or optical photons. The results we have here, therefore, complement and extend the known range of Lorentz factors in GRBs. The values of $A_{\star}$ we find are in the range $2 \times 10^{-4}-3 \times 10^{-3}$ (see Figure 6). We therefore conclude that the expansion occurs into a low-density "wind", having density which is typically 3-4 orders of magnitude below the expectation from a Wolf-Rayet $\operatorname{star}\left(A_{\star} \simeq 0.5-1.0\right)^{28,42,43}$.

Clearly, the fact that a substantial fraction of GRBs have a Lorentz factor of tens rather than hundreds bridges an important observational gap. Other astronomical objects known to have jets such as X-ray binaries or active-galactic nuclei (AGNs) have mildly relativistic jets, with $\Gamma_{i} \lesssim 20$, while earlier estimates of the initial Lorentz factor in GRB jets are in the hundreds. Our result, therefore implies that the range of initial jet velocities that exist in nature does not have a 'gap' in this range $\Gamma_{i}$ of tens, but is rather continuous all the range from mildly relativistic to $\lesssim 1000$. This is shown by the histogram presented in Figure 8.

Since its discovery in early 2005, a plethora of ideas aimed at explaining the X-ray plateau phase appeared in the literature. The first and most commonly used idea is the continuous energy injection from a central compact object which can be a newly formed black hole $8,10,44-47$ or a millisecond magnetar ${ }^{48-54}$. 
Other notable ideas include two components ${ }^{55-58}$ or multi component ${ }^{59}$ jet models; forward shock emission in homogeneous media ${ }^{59}$; scattering by dust/modification of ambient density by gamma-ray trigger $^{60,61}$; dominant reverse shock emission ${ }^{62-66}$; evolving micro-physical parameters ${ }^{46,60}$; and viewing angle effects in which the jets viewed from the off-axis ${ }^{59,67-71}$. While each of these ideas is capable of explaining the observed plateau under certain conditions, they all require an external addition to the basic "fireball" model scenario, and in some cases they cannot address the full set of properties of the plateau phase (such as the flux, slope or duration). A thorough discussion on the advantages and weak points of each of these ideas appear in ${ }^{72}$. We provide a short comparison with some of the recent proposed ideas below in Methods.

Here, we consider a much simpler idea, which does not require any modification of the classical GRB "fireball" model. Rather, we simply look at a different region of the parameter space: a flow having an initial Lorentz factor of the order of few tens, propagating into a "wind" environment, with a typical density of 3-4 orders of magnitude below the expectation from a wind produced by a Wolf-Rayet star. We follow a similar idea that was proposed by Ref. ${ }^{73}$, but did not gain popularity, as (i) the deduced values of the Lorentz factor are lower than the 'fiducial' values, $\Gamma_{i} \gtrsim 100$; and (ii) it was mistakenly claimed that this model can only account for achromatic afterglow, and can therefore explain only a sub-sample of the GRB population ${ }^{72}$. As we showed here, there is no contradiction in the deduced value of the Lorentz factor, and the claim for an achromatic afterglow break is incorrect, as the optical and X-ray bands are not necessary in the same regimes. In our work, we considerably extended this simple idea both theoretically and on the data analysis side. We show that whenever there is enough data to perform a fit in both X-ray and optical bands, the break time in between these two bands is compatible and data in both bands can be interpreted within a single theoretical model. We further carried out a more careful analysis on a much larger data set, allowing larger freedom (more than a single break) and removal of flares on both the X-ray and optical data. We extended the theory to include all possible regimes, thereby showed that different light curves all agree with the same theory. We then showed how this data can be used to infer the values of the density, Lorentz factor, magnetization and fraction of energy carried by the electrons.

While the idea presented in this work is very simple, clearly it has a very far reaching consequences: [a] On the nature of long GRB progenitors, which can either (i) not be a Wolf-Rayet star, or (ii) imply that the properties of the wind ejected by these stars prior to their final explosion is very different than the properties of the wind ejected at earlier times. [b] On our understanding of the nature of the explosion itself, which produce a much wider range of initial jet Lorentz factor, and in many cases are in the range of tens and in others are in the hundreds.

\section{Acknowledgements}

We wish to thank Dr. Damien Bégué for enlightening conversations throughout the project. This work made use of data supplied by the UK Swift Science Data Centre at the University of Leicester. A.P. is supported by the European Research Council via ERC consolidating grant 773062 (acronym O.M.J.). F.R. is supported by the Göran Gustafsson Foundation for Research in Natural Sciences and Medicine. We acknowledge support from the Swedish National Space Agency (196/16), the Swedish Research Council (Vetenskapsrådet, 2018-03513), and the Swedish Foundation for international Cooperation in Research and Higher Education (STINT, IB2019-8160).

\section{Author contributions statement}

H.D.B., A.P. and F.R. wrote the manuscript. H.D.B. has performed sample selection and temporal analysis of the data, theoretical calculations and interpretation. A.P. provided theoretical calculations, interpretation and insight. F.R. assists in the sample selection, interpretation and insight of the results. S.R.O. provides 
the Swift-UVOT count rate light curves and assists in the correction and conversion processes of the data. S.R.O. also enlighten us with her questions and comments. M.G.D. provides the X-ray sample of 222 GRBs and the optical sample of 102 GRBs with all required parameters and assists in the discussion of those two samples. The parameters of those samples are used for initial discussion. M.G.D. assists in the general discussion of the paper as well as the discussion of the Fermi-LAT paper with flat phase. B.Z. assists in the theoretical calculations, discussion and representation of the results. All authors reviewed the manuscript.

\section{Additional information}

Correspondence and requests for materials should be addressed to Hüsne Dereli-Bégué at husnedereli@gmail.com

\section{Competing interests}

The authors declare no competing financial interests.

\section{Data Availability}

The data are publicly available in the literature which are cited in the text. The processed data that support the findings of this study are available from the corresponding author upon reasonable request. The X-ray and the optical light curves of 13 GRBs with the overlaid fit parameters will be available online.

\section{Methods}

\section{Sample and data analysis}

We use the sample of 222 GRBs with plateau phase defined in, Ref. ${ }^{12}$ (see also Refs. ${ }^{19,74-76}$ ). These GRBs were detected by the Neil Gehrels Swift Observatory ${ }^{18}$ from January 2005 until August 2019 with known redshifts ${ }^{1}$. They represent the $56 \%$ of GRBs (394) with known redshifts observed by the Swift satellite in this period. To define this sample, two criteria are used by Ref. ${ }^{12}$, the X-ray light curve of GRBs must have at least one data point in the beginning of the plateau phase and a plateau angle smaller than $41^{\circ}$. The choice of the $41^{\circ}$ comes from the analysis performed in Refs. ${ }^{77,78}$ to allow a more reliable identification of the plateau itself. The plateau phase is identified by fitting the Swift-BAT and XRT data together with the phenomenological Willingale model $^{79}$.

In order to make our analysis as reliable as possible, we limit the burst used to the ones having the best quality observations. Therefore, we added three further criteria to the ones used by Ref. ${ }^{12}$. We require (i) a long lasting (from $10^{2}$ to $10^{5} \mathrm{~s}$ ) plateau phase with a temporal X-ray slope smaller than -0.7 , followed by a power-law decay phase at later times (the self-similar phase). (ii) Sufficient number of data points ( $\gtrsim 5$ which correspond to the number of parameters used to fit the data with one break) during the plateau and self-similar phases to enable the fits to give well constrained parameter values. For this to be valid, we excluded all X-ray flares (see Ref. ${ }^{10}$ figure 1 there in) ${ }^{2}$ defined in the online $S$ wift repository ${ }^{3}$ from the analyzed data. When, after removing the flares we end up having too few data points either during the plateau or the self-similar phase, we excluded the GRB from the analysis.

\footnotetext{
${ }^{1}$ https: //www.mpe.mpg.de/ jcg/grbgen.html

${ }^{2}$ Flares share many properties with prompt emission pulses. They are mainly linked to the late central engine activities ${ }^{10,80}$. They cause steeper slope during both plateau and self-similar phase ${ }^{81-85}$. Moreover, Ref. ${ }^{86}$ show that flares can not be produced in the forward shock.

${ }^{3}$ https: //www.swift.ac.uk/xrt_live_cat/
} 
After doing this, we ended up with 130 GRBs having a well-defined X-ray plateau emission that is followed by a power-law decay phase (the late afterglow, or self-similar phase).

Our last criteria is to have an optical counterpart at around the same time as X-ray data. We find 24 GRBs in Ref. ${ }^{19}$ consistent with these criteria. However, we only have full access to the published optical data. Therefore, we carried out the application of the theoretical model for a representative sample consist of 13 GRBs listed in Table 1, 2, 3 .

\section{$X$-ray data and fitting processes}

The X-ray light curves (LCs) ${ }^{87}$ have been downloaded from the online Swift repository ${ }^{4}$, and have a signal to noise ratio of $4: 1$ with the Swift-XRT bandpass $\left(\mathrm{E}_{\min }, \mathrm{E}_{\max }\right)=(0.3,10) \mathrm{keV}$. We then fit them with a two or three-segments broken power law (BPL) model (i.e. with one or two break times) depending on the required break during the late afterglow phase (self-similar phase), the latter break generally coincides with a jet break (see the canonical X-ray afterglow light curve ${ }^{5}$ in Ref. ${ }^{10}$, figure 1 there in).

During the fit, we excluded the early steep decay segment and high-level emission bumps following the GRB tails (see Ref. ${ }^{10}$ figure 1 there in) defined in the online Swift repository ${ }^{6}$. In addition, we removed any effect by flares. These kinds of faint flares are not being defined in the online Swift repository, however, they affect the definition of the break time at the end of the plateau phase $\left(\mathrm{T}_{a}\right)$ in individual GRB light curves. Comparing the optical and the X-ray break times we found that the X-ray break time $\left(2.8 \pm 0.6 \times 10^{3} \mathrm{~s}\right)$ in the LC of GRB 091029 is earlier than the optical one. This is in contrast with the theoretical expectation that due to the rise in the cooling frequency the transition time is expected to be earlier in the optical band than in the X-ray band. Therefore, by checking the BAT and XRT unabsorbed flux density light curves at $10 \mathrm{keV}$ obtained from the online Swift burst analyser repository, we define a flare between 1379 - 2421 s in the X-ray LC of GRB 091029. Then we objectively removed this flare in the X-ray LC of this burst. It is also important to note that the flares in the online Swift repository ${ }^{7}$ defined by using the phenomenological Willingale model ${ }^{79}$ where they stated that flare identification are indicative, rather than precise.

It is also known that the data obtained by window timing (WT) mode can be effected more from the high latitude emission than the data obtained by photon counting (PC) mode because the WT mode is usually taken at early times as it measures the flux better when the source is bright (see Ref. ${ }^{17}$ for more explanation). Such light curves (WT+PC mode data) require special attention to see if there is an effect on the slopes. Therefore, we only used PC mode of the detector data even if WT mode data exist (GRB 061121 and GRB 060605). As a result, we obtained X-ray temporal slopes $\left(\alpha_{\mathrm{p}, \mathrm{X}}, \alpha_{\mathrm{A} 1, \mathrm{X}}, \alpha_{\mathrm{A} 2, \mathrm{X}}\right)$ and the time of the transition between the two components, $\mathrm{T}_{\mathrm{a}, \mathrm{X}}$ as well as the time of the jet break $\mathrm{T}_{\mathrm{b}, \mathrm{X}}$.

An example for the fitting process: To visualise the fitting processes, we illustrate in Figure 9 the X-ray count light curve obtained from GRB 060614 by the Swift-XRT instrument (during PC mode) with a three-segments BPL model (i.e. with two break times). To perform the fit, we use a Bayesian analysis tool called emcee ${ }^{8}$. We set up the computation with the 64 MCMC walkers, 100 burn-in periods and the 5000 MCMC steps. We chose these steps taking into account the autocorrelation time. The value of the MCMC steps can slightly change for each GRB depending on the autocorrelation time but the choice

\footnotetext{
${ }^{4}$ https: / / www.swift.ac.uk/xrt_curves /

${ }^{5}$ The canonical X-ray afterglow light curve shows five distinct components: the steep decay phase, which is the tail of prompt emission; the shallow decay phase (or plateau); the normal decay phase; the late steepening phase; X-ray flares.

${ }^{6}$ https: //www.swift.ac.uk/xrt_live_cat/

${ }^{7}$ https://www.swift.ac.uk/xrt_live_cat/docs.php

${ }^{8}$ which is an MIT licensed pure-Python implementation of Goodman \& Weare's Affine Invariant Markov chain Monte Carlo (MCMC) Ensemble sampler ${ }^{88}$
} 
of 5000 steps is the one that guarantees that the autocorrelation time is always taken into account. We employ prior distributions for the fit parameters which are $-5<\mathrm{N}<5 ;-5<\alpha_{\mathrm{p}}<5 ;-5<\alpha_{\mathrm{A} 1}<5$; $-5<\alpha_{\mathrm{A} 2}<5 ; 2<\mathrm{T}_{\mathrm{a}}<4.5 ; \mathrm{T}_{\mathrm{a}}<\mathrm{T}_{\mathrm{b}} ; 4.5<\mathrm{T}_{\mathrm{b}}<6.0$ and $-15<\log \mathrm{V}<12$ where $\mathrm{V}$ is intrinsic scatter of the data points. However, these ranges can change depending on the shape of LCs and unit of the data (e.g., count light curve or AB magnitude). We use the uniform (so-called "informative") priors on each parameter except $\mathrm{V}$ assumed to have a log uniform prior. The fit parameters are presented in Table 8 . The corner plot of the posterior probability distributions of fit parameters and the covariances between the fit parameters are presented in Figure 10.

The temporal fit parameters in the X-ray band obtained for 13 GRBs are presented in the Tables 1, 2, 3 , for different cases; case I, case II, and case III respectively. Definition of these cases are explained in the main text above.

The X-ray spectral slopes $\left(\beta_{\mathrm{p}, \mathrm{X}}, \beta_{\mathrm{A} 1, \mathrm{X}}, \beta_{\mathrm{A} 2, \mathrm{X}}\right)$ of the 12/13 GRBs (except 060729) for the same time range as the temporal slopes are obtained from the same online Swift repository ${ }^{9}$ and presented in Tables $1,2,3$. It is important to note that the photon index (which is equal to $\beta+1$ where the $\beta$ is spectral index) is presented in the online Swift repository. For the GRB 060729, we use the SED result from Ref. ${ }^{89}$ since the spectral fit result was not present in the online Swift repository. These X-ray spectral and temporal parameters are combined into the closure relation $\left(F_{v} \propto t^{-\alpha} v^{-\beta}\right)$ in Figure 2 and 3 together with temporal slopes obtained from the temporal fit.

\section{Cautions for the X-ray fit results of a few GRBs:}

- GRB 060605: The X-ray LC has a flare at the beginning of the plateau phase between $350 \mathrm{~s}$ and $600 \mathrm{~s}$ which is not defined in the online Swift repository but it is clearly seen in flux density LC from the burst analysis repository ${ }^{10}$. However, due to the lack of the data points at the end of the plateau phase it is not possible to remove this flare. Because we either end up fitting only the late afterglow (self-similar) data or fitting the data with only one data point at the beginning of the plateau phase which causes unconstrained fit parameters. Therefore, we present the result with this flare in Table 3 , however, we think that this flare effects the optical data more than the X-ray data (see Method 'Optical data' below).

- GRB 080607: Fit results in Table 1 show that this LC has a flat slope with larger errors. This is due to lack of the data points at the end of the X-ray plateau phase. The slope after the plateau phase is quite steep, however, later (last) slope is more compatible with what is expected from the self-similar phase. It is important to mention that this LC has two flares (between 86.0-98.8 s and 114-187 s respectively) defined in the online Swift repository before the plateau phase. Slopes $\left(-2.27_{-0.07}^{+0.07}\right.$ and $-1.42_{-0.30}^{+0.25}$ with a break at $341_{-28}^{+31} \mathrm{~s}$ ) after these flares are very similar to the slopes found after the plateau phase. Therefore, either another flare might exist during this lack of the data or the plateau slope might be steeper with later break than $\mathrm{T}_{\mathrm{a}, \mathrm{X}}$ defined here.

- GRB 130831A: The lack of data in the X-ray LC affects the definition of $T_{a, X}$. We tried to fit the light curve with two breaks, and found that $\log \mathrm{T}_{\mathrm{a}, \mathrm{X}}=3.08_{-0.18}^{+0.28} \mathrm{~s}$ in this case. This break is consistent with being achromatic in both X-ray and optical bands (see Method 'Optical data' below). However, due to the large error bars, caused by the few data points exist during the plateau phase, we found that fitting the light curve with a single break provides better fit, and we therefore

\footnotetext{
${ }^{9}$ https: //www.swift.ac.uk/xrt_live_cut/

${ }^{10}$ https: //www.swift.ac.uk/burst_analyser
} 
used the value presented in Table 1 . In addition, we also define a faint flare between $595-811 \mathrm{~s}$ which coincides with an optical flare and is not defined in the online Swift repository. The result without this flare show that the both slopes $\left(-0.80_{-0.03}^{+0.03}\right.$ and $-0.98_{-0.06}^{+0.06}$ respectively with a break at $\left.1.0_{-0.16}^{+0.25} \times 10^{4} \mathrm{~s}\right)$ are consistent with the expected slope $(\sim-1.2)$ in a self-similar phase. This result is also consistent with a previous study in Ref..$^{90}$. We conclude that either this GRB is not the best representation of the GRBs that show plateau phase or the lack of the data is misleading the results.

These three above examples clearly show that both the flare and lack of the data definitely affect the slopes and definition of the break (e.g. $\mathrm{T}_{\mathrm{a}, \mathrm{X}}$ ) during the temporal fitting.

\section{Optical data and fitting process}

We collected the optical data from the literature. Whenever we found published optical data of an individual GRB (091029, 110213A, 130831A, 171205A) obtained with different instruments we use it in our analysis and performed the temporal fitting simultaneously in each band. Our aim here to reduce the dependence on a single instrument and a single band. Details for each GRB are as follows:

- GRB 091029: The photometric data set obtained in Ref. ${ }^{91}$ in their tables 5, 6, 7, 8, 9. Since all the data were presented in the $\mathrm{AB}$ magnitude, we used it in performing the fit. We fit the data between 427.7 and $2 \times 10^{5} \mathrm{~s}$ with a three-segment broken power law model (i.e. with two break times). The start time corresponds to the peak time of the optical LC, and the first break is at $3.7_{-0.2}^{+0.3} \times 10^{3} \mathrm{~s}$. This break marks the start of the plateau phase. The early (before the plateau) slope is $-0.60_{-0.01}^{+0.02}$. The other fitted parameters, including the second break time and the other slopes are given in Table 1. The end time of our analysis is set by flattening of the optical data (in U-band), likely caused by an external source such as the host galaxy. To interpret the parameters in the $v F_{v}$ space, we multiply these slopes by a constant $1 / 2.5^{11}$. We followed the same procedure for the other GRBs listed below after converting the magnitude to an $\mathrm{AB}$ magnitude if it is not already done.

- GRB 110213A: The photometric observations are obtained from Ref. ${ }^{92}$ in their table 4. This GRB has two peaks in its optical light curve, a main peak is at $263 \mathrm{~s}$ and a secondary peak is at $4827 \mathrm{~s}$. When calculating the slope during the plateau phase, we neglect the contribution from the second peak, which we interpret as a small flare (reverse shock). This flare can also be seen the BAT and XRT unabsorbed flux density light curves at $10 \mathrm{keV}$ in the the online Swift burst analyser repository. This flare cause rising slope during the X-ray plateau phase, however, it has a negligible effect on the the definition of break time at the end of the plateau phase. The fit parameters are presented in Table 1.

- GRB 130831A: The photometric data of the afterglow of this burst is obtained from the VizieR Online Data Catalog 93 . We removed the optical data between $425-3400$ s since we found that the temporal slopes before and after this flare are equal. The end time of our analysis is set by the flattening of the light curves in Rc and Ic bands (this flattening is seen shortly after in all bands) likely caused by an external source such as the host galaxy. After doing so, the end time is at $1.03 \times 10^{5} \mathrm{~s}$ which we find to be similar to the end of the X-ray data. The fit parameters are presented in Table 1.

- GRB 171205A: The photometric data-set are obtained from the online link given in Ref. ${ }^{94}$. Since this GRB is associated to the SN 2017iuk, the optical data started to rise at $\mathrm{T}_{\mathrm{b}, \mathrm{O}}=1.73 \times 10^{5} \mathrm{~s}$

\footnotetext{
${ }^{11}$ This constant comes from the magnitude to the flux conversion formula: $m_{A B}=-2.5 \log f_{v}-(48.585 \pm 0.005)$
} 
( $\approx 2$ days). We fit all the data before the peak of the supernovae $(\mathrm{SN})$ at $9.51 \times 10^{5} \mathrm{~s}$ with two breaks: the first break $\left(\mathrm{T}_{\mathrm{a}, \mathrm{O}}\right)$ represents the transition from plateau to self-similar and the second break, at $T_{b, O}$ marks the rise of the optical light curve due to the supernovae contribution. The fit parameters are presented in Table 2. Caution for the fit results: We find the obtained slopes to be somewhat shallower (or slightly rising) than in other bursts. We believe that this may be due to some contribution from the SN. Similarly, the break time $\mathrm{T}_{\mathrm{a}, \mathrm{O}}$ occurs quite early in the optical band than the X-ray band.

We further use the optical data from Ref. ${ }^{89}$ (their table 8) for the data of GRBs 050319, 060605, 080607, 100418A. In that paper, they also collect all data available in the literature, obtained with different instruments. In our analysis, we excluded data of GRB 100418A since it was reported only in the GCN (Gamma-ray Coordination Network) and no detailed data reduction is available.

- GRB 050319: We fit all the data excluding the last two data points detected after $5.34 \times 10^{5} \mathrm{~s}$ which corresponds to the end of the X-ray data. At this time there is a possible break in the optical band, leading to a slope steeper than -1.2 at later times. However, it is difficult to draw any firm conclusion with only two data points. Even though we fit all the data with two temporal breaks before that time, we only present the plateau and self-similar phase slopes with a single break in Table 3, because the data at early times are from before the beginning of the X-ray plateau phase. In addition, we find that differences between these three slopes are quite small: early (before the plateau) slope is $-0.41_{-0.01}^{+0.02}$, plateau slope is $-0.59_{-0.01}^{+0.01}$ and the slope during the self-similar expansion is $-0.64_{-0.01}^{+0.02}$. The first break time, $371_{-28.6}^{+31.6} \mathrm{~s}$ is too early to be considered as the end of the plateau phase. Moreover, fitting only data obtained by Rc and Ic bands (most of the data obtained in these bands) does not change the result.

- GRB 060605: We fit the data between $185 \mathrm{~s}$ and $2 \times 10^{4} \mathrm{~s}$ with two temporal breaks. The start time of the fit coincides with the peak of the optical bump in the optical data, and it further coincides with the beginning of the X-ray data. The end time corresponds to the time at which the optical data flattens in all bands. Caution for the fit results: In Table 3, we only present the second (last) break time and two slopes interpreted as plateau and self-similar slopes since the slope at early times $\left(0.18_{-0.02}^{+0.01}\right)$ and the first break, at $586_{-43}^{+31} \mathrm{~s}$ (the beginning of plateau phase) correspond to a faint flare in the X-ray data (see more information above in Method 'X-ray data').

- GRB 080607: This LC has three breaks at $89 \mathrm{~s}, 793_{-64}^{+77} \mathrm{~s}$ and $T_{a, O}=2.55_{-0.12}^{+0.26} \times 10^{3} \mathrm{~s}$. We fit the data starting at $89 \mathrm{~s}$ with the two later breaks because we are interested in late time evolution. In Table 3, we present the last break $\left(\mathrm{T}_{\mathrm{a}, \mathrm{O}}\right)$ and last two slopes. Caution for the fit results: The slope before the plateau phase is $-1.22_{-0.02}^{+0.01}$ which is similar to the slope during the self-similar phase. The plateau phase in the optical band is clearly defined with complete data-set and more reliable than the one seen in the X-ray band due to the lack of data (see more information above in Method 'X-ray data').

The optical LC of the rest of the GRBs $(060614,060714,060729,061121,080310)$ in our sample are taken from Ref. ${ }^{7}$ (see Ref. ${ }^{95}$ for the details). These data were only obtained by the Swift Ultraviolet/Optical (UVOT) instrument: V, B, U, W1, M2 and W2. In Ref. ${ }^{7}$, all the LCs were normalized to the U band when analyzing the data.

- GRB 060614: We fit the data in the time range $4.84 \times 10^{3}-8.39 \times 10^{5} \mathrm{~s}$. The LC at early times $(113.16$ - 379.87 s) has a steep decay which coincide by the steep decay in X-ray data in WT mode 
and is therefore interpreted as the end of the prompt phase. At later times $\left(>8.39 \times 10^{5} \mathrm{~s}\right)$ the LC flattens which is interpreted as host galaxy contribution. The fit parameters are presented in Table 2.

- GRB 060714: We exclude the last data point (at $1.49 \times 10^{7} \mathrm{~s}$ ) from the fit since it is a clear outlier. With 8 data points before this last one it is clear that there is a break where $T_{a, X}$ is defined and the parameters are constrained. Including this last data point would be misleading the fit results. It is also important to note that the data points after $10^{5} \mathrm{~s}$ have huge errors. Moreover, we also did not include the negative count rates with larger errors, because they only show no detected in that time range. The fit parameters are presented in Table 1.

- GRB 060729: We fit the data in the time range $266-1.12 \times 10^{6} \mathrm{~s}$. At earlier times, the optical flux rises, and at later times it flattens with a possible break at $6.16_{-0.03}^{+0.02}$, and the slope $-0.16_{-0.07}^{+0.07}$. The fit parameters are presented in Table 2.

- GRB 061121: We fit the data in the time range $104.97-1.66 \times 10^{5} \mathrm{~s}$. The LC at early times $(69.19-89.97 \mathrm{~s})$ has optical flare which coincides with the X-ray flare defined in the online Swift repository, and at later times it flattens. We further exclude the early optical bump seen in the time range 143.45 - $568.48 \mathrm{~s}$ to avoid the miss identification of the plateau slope and the break time $\mathrm{T}_{\mathrm{a}}$. If our fit would include the decay of this bump (namely, starting at $230 \mathrm{~s}$ ), we would obtain decaying slope $-1.01_{-0.06}^{+0.05}$, with a break time at $\log \mathrm{T}=3.12_{-0.06}^{+0.06} \mathrm{~s}$. However, we would find that the later slopes $\left(-0.45_{-0.05}^{+0.06},-0.93_{-0.08}^{+0.07}\right.$ respectively) and the later break time $\left(\log \mathrm{T}_{\mathrm{a}}=4.08_{-0.10}^{+0.10} \mathrm{~s}\right)$ would be compatible with the fit parameters presented in Table 3.

- GRB 080310: We exclude the first data point (at 132.39 s) from the fit because it is an outlier (it is way below the other data points during the plateau phase). This data can be part of the rise at early time in the optical data. Such a rise is clearly seen in some of the optical LC in our sample (e.g., GRB 060605). Moreover, we also did not include the negative count rate with larger error, because it only show no detection in that time. The fit parameters are presented in Table 2.

Optical spectral slopes $\left(\beta_{\mathrm{p}, \mathrm{O}}, \beta_{\mathrm{A} 1, \mathrm{O}}, \beta_{\mathrm{A} 2, \mathrm{O}}\right)$ of $12 / 13$ GRBs in our sample (all except GRB 130831A) are obtained from the literature: data of GRBs 050319, 060605, 060614, 060729, 061121, 080310, 080607 and 100418A are presented in Ref. ${ }^{89}$; GRB060714 in Ref. ${ }^{96}$; GRB091029 in Ref. ${ }^{91}$; GRB110213A in Ref. $^{92}$; and GRB171205A in Ref. ${ }^{94}$. We present these slopes in Tables 1, 2, 3 for the different cases; case I, case II, case III respectively. These spectral slopes are also combined into the closure relation $\left(F_{v} \propto t^{-\alpha} v^{-\beta}\right)$ in Figure 2 and 3 together with temporal slopes obtained from the optical temporal fit.

\section{Flux Ratio}

X-ray energy flux $\left(v F_{V}\right)$ : Unabsorbed flux density $\left(F_{V}\right)$ light curves of the BAT and XRT at $10 \mathrm{keV}^{17}$ are obtained from the online Swift burst analyser repository ${ }^{12}$. These flux density LCs have already been corrected for absorptions from both the galactic and host galaxy. In our analysis, we convert these light curves from flux density (Jy) to energy flux $\left(\mathrm{erg} \mathrm{cm}^{-2} \mathrm{~s}^{-1}\right)$ at $10 \mathrm{keV}\left(v=2.42 \times 10^{18} \mathrm{~Hz}\right)$. Then we used these energy flux LCs to compute $v F_{V}$ X-ray flux at the end of the plateau phase $\left(\mathrm{T}_{\mathrm{a}, \mathrm{X}}\right)$ and at a particular time (typically, $1000 \mathrm{~s})$.

Optical energy flux $\left(v F_{v}\right)$ : In computing the energy flux in the optical band, all the LCs are corrected for both galactic and host galaxy extinctions. i) The galactic extinction along the line of sight are obtained

\footnotetext{
${ }^{12}$ https://www.swift.ac.uk/burst_analyser/
} 
from the IRSA webpage ${ }^{13}$ for $U$ band-pass using the method introduced in Ref. ${ }^{97}$. ii) The extragalactic reddening $\mathrm{E}(\mathrm{B}-\mathrm{V}$ ) of all GRBs (except GRBs 060714 and 100418A) are obtained during the plateau phase and the references are listed in Table 9. We first used these values to compute the host galaxy extinctions in the V-band (except for 060714 which has $A_{v}$ in Ref. ${ }^{98}$ ). Second, we use these extinctions and the method introduced in Ref. ${ }^{99}$ to compute the host galaxy extinction in $\mathrm{U}$ band. Both extinctions (galactic and host galaxy) for each GRBs are presented in Table 9. Note that flux density of four GRBs $(050319,060605,080607,100418 \mathrm{~A})$ taken from Ref. ${ }^{89}$ are already corrected for both galactic and host extinction in that paper.

- For six GRBs (060614, 060714, 060729, 061121, 080310 and 091029), the optical count rate LCs from Ref. $^{7}$ are used to compute the U band energy flux LCs. For the conversion, we used the U band count rate to flux conversion factor $\left(1.628 \times 10^{-16} \mathrm{erg} \mathrm{cm}^{-2}\right.$ Angstrom $^{-1}$ counts $\left.^{-1}\right)$ provided in the UVOT calibration webside ${ }^{14}$ and labelled as count rate to flux ratio in table 2 there in. Then we computed the energy flux $\left(v F_{v}\right)$ LCs, where $v$ is taken at the U-band $\left(8.65 \times 10^{14} \mathrm{~Hz}\right)$. These U-band energy flux LCs are used to compute the $v F_{v}(\mathrm{U})$ optical flux at $\mathrm{T}_{\mathrm{a}, \mathrm{X}}$ and at a given time $\left(\sim 1000 \mathrm{~s}\right.$ which is called a reference time $\left.\mathrm{T}_{\text {ref.,U}}\right)$.

For the GRBs which are not presented in Ref. ${ }^{7}$ we followed a different procedure:

i) For the GRBs in Ref. ${ }^{89}(050319,060605,080707,100418)$, the $v F_{v}$ optical flux at the end of the X-ray plateau phase $\left(\mathrm{T}_{\mathrm{a}, \mathrm{X}}\right)$ are obtained from the flux density LC in table 7 of the online material in Ref. ${ }^{89}$. In these cases, the frequency $v$ is different for each GRBs (V band $=5.48 \times 10^{14} \mathrm{~Hz}, \mathrm{CR}$ and $\mathrm{RC}$ bands $=4.55 \times 10^{14} \mathrm{~Hz}$, white band $=7.79 \times 10^{14} \mathrm{~Hz}$, see Table 9 below). For consistency, whenever possible we used the U-band in calculating the LC. When U-band data was not available, we used other bands. We do note that in such cases, the differences between different optical bands $\left(\sim 10^{15} \mathrm{~Hz}\right)$ are quite small compared to the X-ray band $\left(\sim 10^{18} \mathrm{~Hz}\right)$.

ii) For the other GRBs not presented in $\operatorname{Ref}^{89}$ (110213A, 130831A, 171205A), we followed the same procedure as in appendix A of Ref. ${ }^{89}$ to convert the optical data (AB magnitude) to the optical energy flux $\left(v F_{v}\right)$ light curve in the $\mathrm{U}$ band.

Energy flux ratio: Both $v F_{v}(\mathrm{X})$ and $v F_{v}(\mathrm{U})$ fluxes at $\mathrm{T}_{\mathrm{a}, \mathrm{X}}$ are used to compute the energy flux ratio between X-ray and optical bands, and are presented in Table 7.

Isotropic energy: The Swift-BAT observations are carried in a relatively narrow energy range (15-150 $\mathrm{keV}$ ). Thus, to compute the isotropic energy $\mathrm{E}_{\mathrm{iso}}$, we followed the same method presented in Ref. ${ }^{100}$. In this method, a simple power-law spectral model adopted i.e., $\phi(E)=E^{\alpha_{\gamma}}$, where $\phi(E)$ is the source photon spectrum and $\alpha_{\gamma}$ is the photon spectral index, to calculate the isotropic-equivalent energy, $\mathrm{E}_{\text {iso }}$ taking into account the $k$-correction:

$$
\mathrm{E}_{\mathrm{iso}}=\frac{4 \pi \mathrm{d}_{\mathrm{L}}^{2} \mathrm{~S}}{(1+\mathrm{z})^{3-\alpha_{\gamma}}} .
$$

where, $S$ is BAT fluence (15-150 keV). Both $S$ and $\alpha_{\gamma}$ are taken from the $S$ wift GRB table ${ }^{15}, \mathrm{~d}_{\mathrm{L}}(\mathrm{z})$ is the luminosity distance calculated assuming a flat $\Lambda \mathrm{CDM}$ cosmological model with cosmological parameters $\Omega_{m}=0.286$ and $\mathrm{H}_{0}=70 \mathrm{~km} \mathrm{~s}^{-1} \mathrm{Mpc}^{-1}$ in CosmoCalc ${ }^{16}$, and $z$ is the redshift.

\footnotetext{
${ }^{13}$ https://irsa.ipac.caltech.edu/applications/DUST/

14https://heasarc.gsfc.nasa.gov/docs/heasarc/caldb/swift/docs/uvot/index.html

15 https://swift.gsfc.nasa.gov/archive/grb_table/

${ }^{16}$ http: //www.astro.ucla.edu/ wright/CosmoCalc.html ${ }^{101}$
} 
The various parameters relevant to our study are listed in Table 6: (i) the GRB name, (ii) its redshift $(z)$, (iii) the luminosity distance $\left(\mathrm{d}_{\mathrm{L}}\right)$, (iv) fluence $(S)$, (v) photon spectral index $\left(\alpha_{\gamma}\right)$, (vi) isotropic energy $\left(\mathrm{E}_{\mathrm{iso}}\right)$, (vii) the time at the end of the X-ray plateau phase $\left(\mathrm{T}_{\mathrm{a}, \mathrm{X}}\right)$, (viii) $v F_{v} \mathrm{X}$-ray flux and (ix) $v F_{v}$ optical ( $\mathrm{U}$ band) flux at $\mathrm{T}_{\mathrm{a}, \mathrm{X}},(\mathrm{x})$ a reference time in the optical band called $\mathrm{T}_{\text {ref., } \mathrm{U}}$ (typically at around $1000 \mathrm{~s}$ ), (xi) $v F_{v} \mathrm{X}$-ray flux and (xii) $v F_{v}$ optical (U band) flux at the reference time $\mathrm{T}_{\text {ref., } \mathrm{U}}$. The isotropic energy $\left(\mathrm{E}_{\text {iso }}\right)$ and a time at the end of the X-ray plateau phase $\left(\mathrm{T}_{\mathrm{a}, \mathrm{X}}\right)$, firstly discovered by Ref. ${ }^{102-104}$, are presented in Figure 1.

\section{Theoretical model}

The key to understanding the observed signal is the realization that the end of the plateau corresponds to the transition from a coasting phase to a self-similar (decaying) expansion phase of the expanding plasma. The observed signal originates entirely from ambient electrons collected and heated by the forward shock wave, propagating at relativistic speeds inside a "wind" (decaying density) ambient medium. During the transition from the coasting to the decaying phases a reverse shock crosses the expanding plasma. However, contribution from electrons heated by the reverse shock is suppressed due to (i) the declining ambient density which implies that the ratio of plasma density to ambient density remains constant (in a conical expansion), and (ii) its slower speed, which translates into less energetic electrons that emit at much lower frequencies than forward shock heated electrons, implying that the contribution to the optical and X-ray bands is negligible.

To understand how this transition affects the observed spectra, we first describe the radiative mechanism adopted, which is the classical synchrotron emission from a power-law distribution of electrons, assuming a power law index $p$, namely $N_{e l}(\gamma) d \gamma \propto \gamma^{-p}$ above a minimum value $\gamma_{m}$; below this value, it is safe to assume that the electrons have a Maxwellian (or quasi-Maxwellian) energy distribution ${ }^{13,20}$. This assumption leads to a broken power law spectrum, whose shape, in the relevant observed bands (frequency $v$ ), depends on whether the peak frequency, $v_{m}$ (defined in Equation 4) is above or below the cooling frequency, $v_{c}$ (defined in Equation 5). At low frequencies, one needs to consider the self-absorption frequency, which is neglected here being below the optical frequency.

For $v_{m}>v_{c}$ (the so-called "fast cooling" regime, expected at early times), the possibilities (marked $[\mathrm{A}]-[\mathrm{C}])$ are

$$
F_{v}=F_{v_{\max }} \times\left\{\begin{array}{lll}
v_{c}^{-1 / 3} v^{1 / 3} & v<v_{c}<v_{m} & {[A]} \\
v_{c}^{1 / 2} v^{-1 / 2} & v_{c}<v<v_{m} & {[B]} \\
v_{m}^{(p-1) / 2} v_{c}^{1 / 2} v^{-p / 2} & v_{c}<v_{m}<v & {[C]}
\end{array}\right.
$$

At later times, $v_{m}<v_{c}$ and the plasma enters the "slow cooling" regime, in which (regions [D]-[F])

$$
F_{v}=F_{v_{\max }} \times\left\{\begin{array}{lll}
v_{m}^{-1 / 3} v^{1 / 3} & v<v_{m}<v_{c} & {[D]} \\
v_{m}^{(p-1) / 2} v^{-(p-1) / 2} & v_{m}<v<v_{c} & {[E]} \\
v_{m}^{(p-1) / 2} v_{c}^{1 / 2} v^{-p / 2} & v_{m}<v_{c}<v & {[F]}
\end{array}\right.
$$

Here, $v_{m}$ is the typical emission frequency from electrons at the peak of the distribution, namely having Lorentz factor $\gamma_{m}$, and is given by (for an on-axis observer)

$$
v_{m}^{o b}=\frac{3}{4 \pi} \frac{q B}{m_{e} c} \gamma_{m}^{2} \frac{\Gamma}{(1+z)}=4.196 \times 10^{6} B \gamma_{m}^{2} \frac{\Gamma}{(1+z)} \mathrm{Hz},
$$

where $m_{e}$ is the electron's mass, $c$ is the speed of light, $B$ is the magnetic field, $\Gamma$ is the bulk motion Lorentz factor and $z$ is the redshift. The cooling frequency $v_{c}$ is the frequency of emission from electrons whose 
radiative cooling time is equal to the dynamical time,

$$
v_{c}^{o b}=\frac{3}{4 \pi} \frac{q}{m_{e} c}\left(\frac{6 \pi m_{e} c^{2}}{\sigma_{T}}\right)^{2} \frac{\Gamma^{3}}{B^{3} r^{2}(1+z)}=2.26 \times 10^{45} \frac{\Gamma^{3}}{B^{3} r^{2}(1+z)} \mathrm{Hz} .
$$

Here, $q$ is the electron's charge, $\sigma_{T}$ is Thomson's cross section, and $r$ is the plasma radius, which is related to the dynamical time (in the comoving frame) by $r \sim \Gamma c t_{\text {dyn }}{ }^{17}$. The peak flux is estimated by $F_{v, p e a k}^{o b}=\frac{1}{4 \pi d_{L}^{2}} N_{e} P_{v, \text { max }}^{o b}$, where $d_{L}$ is the luminosity distance, $N_{e}$ is the number of radiating particles and $P_{v, \text { max }}^{o b}=P_{\text {tot }}^{o b} / v_{\text {peak }}^{o b}$, where $P_{\text {tot }}^{o b}$ is the total power radiated by synchrotron emission, and $v_{\text {peak }}^{o b}=v_{m}^{o b}$. This gives

$$
P_{v, \text { max }}^{o b}=\frac{P_{t o t}^{o b}}{v_{m}^{o b}}=\frac{\frac{4}{3} c \sigma_{T} \gamma_{m}^{2} \frac{B^{2}}{8 \pi} \Gamma^{2}}{\frac{3}{4 \pi} \frac{q B}{m_{e} c} \gamma_{m}^{2} \frac{\Gamma}{(1+z)}}=\frac{2}{9} \frac{m_{e} c^{2} \sigma_{T}}{q} B \Gamma(1+z)=2.53 \times 10^{-22} B \Gamma(1+z)
$$

We assume that all the ambient particles collected by the forward shock wave radiate. By assumption, these mass density is given by $\rho(r)=A / r^{2}$, implying that

$$
N_{e}(r)=\frac{4 \pi}{m_{p}} \int_{0}^{r} \rho\left(r^{\prime}\right) r^{\prime 2} d r^{\prime}=\frac{4 \pi A r}{m_{p}}
$$

The proportionality constant $A$ is calculated assuming that prior to its final explosion, the progenitor star ejects mass at a constant rate and at a constant velocity, resulting in $\rho(r)=n(r) m_{p}=\frac{\dot{M}}{4 \pi v_{w} r^{2}} \equiv A r^{-2}$. For a Wolf-Rayet progenitor, the typical values are ${ }^{105}, \dot{M}=10^{-5} M_{\odot} \mathrm{yr}^{-1}$, and wind velocity $v_{w}=10^{8} \mathrm{~cm} \mathrm{~s}^{-1}$. These values lead to $A=5 \times 10^{11} A_{\star} \mathrm{gr} \mathrm{cm}^{-1}$.

\section{Dynamics, magnetic field and electron's energy.}

Following an initial acceleration phase, the plasma coasts at $\approx$ steady Lorentz factor $\Gamma_{i}$. Once it collects sufficient material from the ambient medium, $m_{I S M} \gtrsim m_{i} / \Gamma_{i}$ where $m_{i}$ is the initial ejected mass, the flow becomes similar, and its evolution is described by the well-known self-similar solution ${ }^{15}$. For an instantaneous explosion releasing energy $E$ that occurs into a density gradient this solution reads

$$
\Gamma(E ; r)=\left(\frac{9 E}{16 \pi \rho(r) c^{2} r^{3}}\right)^{1 / 2}=\left(\frac{9 E}{16 \pi A c^{2} r}\right)^{1 / 2}
$$

The relation between the Lorentz factor, radius and observed time in this case was calculated by Ref. ${ }^{106}$, $t^{\mathrm{ob}} \simeq(1+z) r /\left(2 \Gamma^{2}(r) c\right)$, enabling to express the Lorentz factor as a function of the observed time during this phase,

$$
\Gamma\left(E, A ; t^{\mathrm{ob}}\right)=\left(\frac{9 E(1+z)}{32 \pi A c^{3} t^{\mathrm{ob}}}\right)^{1 / 4}
$$

Transition from the initial (coasting) to the later (self-similar expansion) phases occurs once $\Gamma\left(E, A ; t^{\mathrm{ob}}\right)<$ $\Gamma_{i} 18$. Thus,

$$
t_{\text {trans }}^{\mathrm{ob} .}=(1+z) \frac{9 E}{32 \pi A c^{3} \Gamma_{i}^{4}} .
$$

\footnotetext{
${ }^{17}$ For simplicity, we neglect a possible factor of the order unity, as well as contribution form inverse-Compton (IC) cooling, which is negligible for the parameters used.

${ }^{18} \mathrm{~A}$ second condition is that $t>t_{G R B}$, which is always met.
} 
It is explicitly assumed that both the generation of magnetic field and acceleration of particles to high energies occur at the shock wave. The energy density behind the shock is given by the shock jump conditions, $u \simeq 4 \Gamma^{2} n m_{p} c^{2}$. Therefore, during the initial (coasting) phase where $\Gamma=\Gamma_{i}$, this energy density is $u\left(t^{\text {obs. }}\right)_{\text {coasting }}=\frac{A}{\Gamma_{i}^{2} t_{\text {obs. }}^{2}}(1+z)^{2}$. We adopt the standard assumption that a fraction $\varepsilon_{B}$ of this energy is used in generating a magnetic field, and a fraction $\varepsilon_{e}$ is used in heating (accelerating) the electrons. During the initial (coasting) phase, this gives a magnetic field strength $B_{\text {initial }}=\left(8 \pi \varepsilon_{B} u\right)^{1 / 2}=12(1+$ z) $A_{\star}^{1 / 2} \Gamma_{i, 1.5}^{-1} t_{3}^{\text {obs. }}{ }^{-1} \varepsilon_{B,-2}^{1 / 2} \mathrm{G}$ and typical electron Lorentz factor, $\gamma_{m, \text { initial }}=\varepsilon_{e} \Gamma_{i}\left(\frac{m_{p}}{m_{e}}\right)=5.5 \times 10^{3} \Gamma_{i, 1.5} \varepsilon_{e,-1}$. Here and below $Q_{, x}=Q / 10^{x}$.

During the deceleration phase, a similar calculation with the use of Equation 8 gives

$$
B_{\text {late }}=\left(\frac{2048 \pi^{3} A^{3} c^{3}(1+z)^{3} \varepsilon_{B}^{2}}{9 E t^{\mathrm{ob}^{3}}}\right)^{1 / 4}=0.74\left(\frac{1+z}{2}\right)^{3 / 4} E_{53}^{-1 / 4} A_{\star}^{3 / 4} t_{\mathrm{day}}^{\mathrm{ob}}{ }^{-3 / 4} \varepsilon_{B,-2}^{1 / 2} \mathrm{G}
$$

and

$$
\gamma_{m, \text { late }}=\varepsilon_{e} \Gamma \frac{m_{p}}{m_{e}}=\varepsilon_{e}\left(\frac{m_{p}}{m_{e}}\right)\left(\frac{9 E(1+z)}{32 \pi A c^{3} t^{\mathrm{ob}}}\right)^{1 / 4}=2040\left(\frac{1+z}{2}\right)^{1 / 4} E_{53}^{1 / 4} A_{\star}^{-1 / 4} t_{\mathrm{day}}^{\mathrm{ob}}{ }^{-1 / 4} \varepsilon_{e,-1} .
$$

\section{Temporal and spectral signal.}

Using these results in equations 4, 5 and 6 gives the parametric dependence of the key observed frequencies and flux:

$$
\begin{aligned}
& v_{m}^{\mathrm{ob} .}= \begin{cases}4.6 \times 10^{16} A_{\star}^{1 / 2} \Gamma_{i, 1.5}^{2} t_{3}^{\mathrm{ob} .}{ }^{-1} \varepsilon_{e,-1}^{2} \varepsilon_{B,-2}^{1 / 2} \mathrm{~Hz} & \text { (coasting), } \\
7.2 \times 10^{13}\left(\frac{1+z}{2}\right)^{1 / 2} E_{53}^{1 / 2} t_{\text {day }}^{\mathrm{ob}}{ }^{-3 / 2} \varepsilon_{e,-1}^{2} \varepsilon_{B,-2}^{1 / 2} \mathrm{~Hz} & \text { (decay), }\end{cases} \\
& v_{c}^{\mathrm{ob} .}= \begin{cases}3 \times 10^{12}\left(\frac{1+z}{2}\right)^{-2} A_{\star}^{-3 / 2} \Gamma_{i, 1.5}^{2} t_{3}^{\mathrm{obs} .} \varepsilon_{B,-2}^{-3 / 2} \mathrm{~Hz} & \text { (coasting), } \\
3.7 \times 10^{13}\left(\frac{1+z}{2}\right)^{-3 / 2} E_{53}^{1 / 2} t_{\text {day }}^{\mathrm{ob}}{ }^{1 / 2} \varepsilon_{B,-2}^{-3 / 2} A_{\star}^{-2} \mathrm{~Hz} & \text { (decay), }\end{cases}
\end{aligned}
$$

and

$$
F_{v, \text { peak }}^{\text {ob. }}= \begin{cases}6.1 \times 10^{-24}\left(\frac{1+z}{2}\right) d_{L, 28.3}^{-2} A_{\star}^{3 / 2} \Gamma_{i, 1.5}^{2} \varepsilon_{B,-2}^{1 / 2} \mathrm{erg} \mathrm{cm}^{-2} \mathrm{~s}^{-1} \mathrm{~Hz}^{-1} & \text { (coasting) }, \\ 9.9 \times 10^{-25}\left(\frac{1+z}{2}\right)^{3 / 2} d_{L, 28.3}^{-2} E_{53}^{1 / 2} A_{\star} \varepsilon_{B,-2}^{1 / 2} t_{\text {day }}^{\mathrm{ob}}-1 / 2 \mathrm{erg} \mathrm{cm}^{-2} \mathrm{~s}^{-1} \mathrm{~Hz}^{-1} & \text { (decay) } .\end{cases}
$$

The results of equations 11 and 12 imply that both at early (coasting) and later (self-similar decay) phases, the peak frequency decreases with observed time, while the cooling frequency increases with time. This have two important consequences on the spectral and temporal evolution of the observed signal: (i) At high enough frequencies, the transition always occurs from region $[\mathrm{F}]\left(v_{c}<v\right)$ to region $[\mathrm{E}]\left(v<v_{c}\right) ; 19$ and (ii) the transition always occurs at lower frequencies first- it will occur in the optical band before the $\mathrm{X}$-ray band, regardless of whether the flow is in the coasting or in the decaying phase.

Using these results in Equations 2 and 3 give the temporal and spectral dependence in each of the 6 possible regimes considered, both during the coasting and during the self-similar decay phases (see for consistency with Ref. ${ }^{107}$ ). These are summarized in Table 4.

These results can now be used to explain the observed signal, which is in an excellent agreement with the theoretical model. Provided that the transition to region [E] occurs after the end of the coasting

\footnotetext{
${ }^{19}$ At lower frequencies, it occurs from region [D] to region [E]
} 
phase, the light curve during the coasting phase is $\approx$ flat, $F_{V} \propto t_{\text {obs. }}^{(2-p) / 2} \sim t_{\text {obs. }}^{0.0 .-0.2}$, which is similar to the observed signal in the X-ray band in cases I and II. Since the transition to region [E] occurs earlier at longer wavelength, in some of the cases I the optical band is already in region [E], in which case a decay in the optical light curve, $F_{V} \propto t_{\text {obs. }}^{(1-p) / 2} \sim t_{\text {obs. }}^{-0.5 .-0.7}$ is expected. The only other option is that the transition to region [E] occurs at earlier times, in which case both the $\mathrm{X}$ - and the optical light curves show a decay, which is the relevant scenario for case III here.

Once the flow shifts to the self-similar decay phase, both the X-ray and the optical light curves decay as $F_{V} \propto t_{\text {obs. }}^{(2-3 p) / 4} \sim t_{\text {obs. }}^{-1.0 .-1.3}$ if in region [F], or alternatively $F_{V} \propto t_{\text {obs. }}^{(1-3 p) / 4} \sim t_{\text {obs. }}^{-1.25 .-1.55}$ if in region $[E]$. However, the difference in the temporal index in between these two cases is only $1 / 4$, and may not be easily identified due to the noise.

\section{Determining the physical properties of the outflow.}

A flat X-ray plateau indicates that the flux in the X-ray band is in region [F]. Assuming, for simplicity power law index $p=2$, equation 3 gives

$$
v F_{v}(\mathrm{X})^{\mathrm{ob} .}=F_{v, \text { peak }}^{\mathrm{ob}} v_{c}^{1 / 2} v_{m}^{1 / 2}=\frac{1}{d_{L}^{2}} c^{3} A \Gamma_{i}^{4} \varepsilon_{e}
$$

where we made use of Equations 11, 12 and 13. Combining this result with Equation 10, one finds that for bursts with known redshift, the transition time and X-ray flux provide a direct measurement of $\varepsilon_{e}$ :

$$
\varepsilon_{e}=\frac{d_{L}^{2}}{(1+z)} \frac{32 \pi t_{\mathrm{trans}}^{\mathrm{ob}}}{9 E} \nu F_{v}(\mathrm{X})^{\mathrm{ob}}
$$

Furthermore, the transition time provides a strong constraint on the ambient medium and the initial Lorentz factor. This is done by writing equation 10 in the form

$$
A \Gamma_{i}^{4}=(1+z) \frac{9 E}{32 \pi c^{3} t_{\text {trans }}^{\text {ob. }}},
$$

which is a very robust result.

A further constrain can be put by using the temporal behaviour of the optical data. In case I, the optical light curve decays, namely the optical band is in region [E], and $v F_{v}(U)^{\mathrm{ob}}=F_{v, \text { peak }}^{\mathrm{ob}} v_{m}^{1 / 2} v_{\mathrm{U}}^{1 / 2}$ (for $p=2$ ). Using Equations 11, 13, 15 and using an observed optical band $v_{\mathrm{U}}=8.65 \times 10^{14} \mathrm{~Hz}$, one finds

$$
A_{\star}^{7 / 4} \Gamma_{i, 1.5}^{3} \varepsilon_{B,-2}^{3 / 4}=4 \times 10^{-3} .
$$

Thus, for a given density parameter $\left(A_{\star}\right)$, there is a corresponding magnetic parameter $\left(\varepsilon_{B}\right)$. One may therefore use an external knowledge of $\varepsilon_{B}$ (e.g., $\varepsilon_{B}=1$ ) to completely determine the values of $A_{\star}$ and therefore of $\Gamma_{i}$. We further note that in this case, the ratio of fluxes,

$$
R_{I} \equiv \frac{v F_{V}(\mathrm{X})}{v F_{V}(\mathrm{U})}=\left(\frac{v_{c}}{v_{\mathrm{U}}}\right)^{1 / 2}\left(\frac{v_{\mathrm{X}}}{v_{\mathrm{U}}}\right)^{(2-p) / 2}
$$

is expected in the range $10 \leq R \leq 300$ for power law indices in the range $1.8 \leq p \leq 2.4$.

In case II, the optical light curve is flat, namely it is in region [F]. Equations 15 and 16 are valid, and thus a direct measurement of $\varepsilon_{e}$ and of $A \Gamma_{i}^{4}$ exist. However, as $v_{c}<v_{\mathrm{U}}$, the ratio of the optical and X-ray fluxes is constant,

$$
R_{I I} \equiv \frac{v F_{v}(\mathrm{X})}{v F_{V}(\mathrm{U})}=\left(\frac{v_{\mathrm{X}}}{v_{\mathrm{U}}}\right)^{(2-p) / 2} \approx 1
$$


Instead, we use the fact that $v_{c}$ increases with time (Equation 12) to argue that in this case at the transition time (Equation 10), $v>v_{c}$. This gives a lower limit on the magnetization,

$$
\varepsilon_{B,-2}^{3 / 2}>3.1 \times 10^{-3}\left(\frac{1+z}{2}\right)^{-1} E_{52.5} A_{\star}^{-5 / 2} \Gamma_{i, 1.5}^{-2} .
$$

Thus, in this case, for a given $A_{\star}$ there is a minimum value of the magnetic field, which is inversely proportional to $A_{\star}$. Thus, again, one may use an external constraint (such as $\varepsilon_{B}<1$ ) to constrain a minimum value of $A_{\star}$ which translates to a maximum value of $\Gamma_{i}$.

Finally, in case III both the X-ray and the optical light curves decay, namely both are in region [E]. In this case, Equation 16 is valid, but not Equation 15. It is therefore not possible to obtain a direct measure of $\varepsilon_{e}$ in this case. The best constraint can be put by using the requirement that at the beginning of the plateau, at $\sim 100 \mathrm{~s}$, the $\mathrm{X}$-ray frequency is already below the cooling frequency, $v_{\mathrm{X}}<v_{c}$. This translates into an upper limit,

$$
\left(\frac{1+z}{2}\right) A_{\star}^{3 / 2} \Gamma_{i, 1.5}^{-2} \varepsilon_{B,-2}^{3 / 2}<2.5 \times 10^{-6}
$$

This provides an upper limit on the value of $\varepsilon_{B}$ for a given $A_{\star}$, which increases as $A_{\star}$ decrease. Using external constraint, e.g., $\varepsilon_{B}=0.01$ is therefore useful in providing an upper limit on $A_{\star}$, and a lower limit on $\Gamma_{i}$. Combined with a measurement of the optical flux, this also gives a lower limit on the value of $\varepsilon_{e}$.

The ratio of the X-ray to optical fluxes in this case is intermediate,

$$
R_{I I I} \equiv \frac{v F_{v}(\mathrm{X})}{v F_{v}(\mathrm{U})}=\left(\frac{v_{\mathrm{X}}}{v_{\mathrm{U}}}\right)^{(3-p) / 2} \approx 10 \ldots 150
$$

depending on the value of $p$.

\section{Comparison with ideas of explaining X-ray plateau}

As discussed in the main text, a plethora of models were proposed in the literature in attempts to explain the X-ray plateau. Here we discuss some of the most recent works, focusing on three topics: energy injection, reverse shock, and viewing angle.

Metzger et al. (2011) propose a millisecond protomagnetar model for $\mathrm{GRBs}^{48}$. In this magnetar model, the energy released by dipole radiation is predicted to be transferred to the surroundings via a magnetar wind. This wind is heated by neutrinos just after the launch of the supernova shock from a core collapse of a massive star. The outflow is collimated into a bipolar jet by its interaction with the progenitor star. As the magnetar cools, the wind becomes ultrarelativistic and Poynting flux dominated on a time-scale comparable to that required for the jet to clear a cavity through the star. Therefore, in this model the magnetic dissipation and shocks explain prompt emission and the steep decay phase that follows. The late time flaring or afterglow emission, such as the X-ray plateau are thought to be powered by continuous magnetic or the residual rotational energy. Indeed, Rowlinson et al. (2014) ${ }^{49}$ explain the the correlation between luminosity and duration of the plateau phase within the $1 \sigma$ uncertainties $^{78}$ with this model in which the magnetic field and the spin periods typical of a fast rotating neutron star. A later paper by Rea et al. $(2015)^{50}$ show that the magnetar model can be reconciled within the GRB emission in the plateau only if supermagnetar with high magnetic field strength are allowed.

In a successive paper of Stratta et al. $(2018)^{51}$ for the first time a non-ideal modelling of spindown magnetar is fitted to the afterglow data with a statistical sample of 40 long GRBs with a well-defined 
plateau and 13 short GRBs including the short with extended emission. The conclusion reached in that paper is that short GRBs including the short GRBs with extended emission and long GRBs can be explained within the magnetar model. However, the difference between the samples is that the long GRBs occupy a lower end in the magnetic field, spin period plane compared to the short GRBs. Therefore, the long GRB sample has a lower spin, P, and a lower magnetic field, B. The correlation between magnetic field and spin period follow the established physics of the spin-up line for accreting neutron star (NS) in alactic binary systems. The $B-P$ relation obtained with the sample of 53 GRBs matches spin-up line predictions for the magnetar model with mass accretion rates expected in the GRB prompt phase. The latter are $\sim 11-14$ orders of magnitude higher than those inferred for the galactic accreting NSs.

Matsumoto et al. $2020^{52}$ show a link between extended emission seen in some short GRBs and the plateau phase. They assume a continues energy injection from a central compact object, such as a magnetar, for the origin of both phases of emissions. Such an extended emission lasting up to $10^{2}-10^{3} \mathrm{~s}$ is also reported in several long GRBs ${ }^{108,109}$. In our study, when analyzing the full sample of 222 GRBs with known redshift and a plateau phase, we found that when such an extended emission exists, it affects the slopes of both the plateau and the following self-similar phases. The slopes for those bursts that show extended emission are steeper in both the plateau and self similar phases $(>-0.7$ and $>-1.2$ respectively) than the limit we take as an indication for a plateau (temporal slope of $<-0.7$ ). These are therefore excluded from our final sample.

Zhao et al. $2020^{53}$ argued that in a few cases, there is a steep decay following the plateau phase, after which a second plateau may exist. They interpreted this as an outcome of a central engine consists of a rapidly spinning magnetar that collapses to a newborn black hole. When analyzing the 3 GRBs in their sample, we found that the evidence for a steep decay is not strong: the X-ray LC of these 3 bursts either contain flares, or a very few data points exist.

In contrast to previous works, Çıkıntoğlu et al. (2020) ${ }^{54}$ argue that clear plateau phase may not be realized if the magnetic field of the nascent magnetar is in a transient rapid decay stage. Due to this stage the spin-down power may decline so fast and cause a lack of the plateau phase. With this idea, they analyze the X-ray light curve of 6 GRBs without plateau and conclude that these GRBs might be hosting millisecond magnetars.

A different type of model was proposed by Lyutikov et al. (2017) ${ }^{65}$ and Barkov et al. (2021) ${ }^{66}$, in which the plateau phase, flares and possible steep slopes immediately after the plateau phase, are all explained by a dominant reverse shock. This reverse shock is different than the "classical" reverse shock 20 predicted as part of the "fireball" model ${ }^{62,63}$ because it is assumed to propagate through ultrarelativistic, highly magnetized pulsar-like winds produced by long-lasting central engines. While this is an interesting idea, similar to the models discussed above, only very few GRBs observed until now seem to fit the predictions of this model. Moreover, those GRBs have several flares during their X-ray data which makes it very difficult to draw firm conclusion. Within the realm of the classical "fireball" model, contribution from the reverse shock is expected during the transition from the coasting to the similar phase. Indeed when analyzing the light curves of the GRBs in our sample we did see some evidence for a possible contribution from a reverse shock in the optical light curve (especially in GRB 110213A). However, full analysis of this effect is beyond the scope of this manuscript, and will be presented elsewhere.

Another suggestion, recently promoted by Oganesyan et al. (2019) ${ }^{70}$ and Beniamini et al. (2020) ${ }^{71}$ is that of a structured jet viewed from off-axis. These works were motivated by the observation of an off-axis jet detected from the very low luminosity GRB/GW 170817. While Oganesyan et al. (2019) ${ }^{70}$ explained

\footnotetext{
${ }^{20}$ Such a reverse shock can be easily suppressed by the external forward shock in the X-ray band due to the its faintness at least an order of magnitude lower ${ }^{72}$.
} 
the plateau phase by high-latitude emission, Beniamini et al. (2020) ${ }^{71}$ argued in favour of a structured jet on the near-core lines of sight. Indeed, the addition of degrees of freedom in these models, enable good fits to several bursts which show a plateau.

In this work, we adopted and developed a much simpler idea than all of the above, which does not require any addition or modification to the classical GRB "fireball" model. Instead, we simply consider a different region of parameter space: a flow having an initial Lorentz factor of the order of few tens, propagating into a "wind" environment, as proposed earlier by Ref. ${ }^{73}$. We find that this model can naturally explain the observed plateau, both in the optical and X-rays, within the framework of synchrotron emission from particles accelerated to a power law distribution by the forward shock wave. We further find that these assumptions lead to a typical ambient density of 3-4 orders of magnitude below the expectation from a wind produced by a Wolf-Rayet star. In our work, we show that this simple theoretical idea perfectly matches with many observations obtained in both X-ray and optical bands. We further extended the theory to show how it can be used to extract meaningful information on the outflow properties, and showed that there is no contradiction between the values found here and those known from GRBs which do not show a plateau.

\section{References}

1. Krolik, J. H. \& Pier, E. A. Relativistic motion in gamma-ray bursts. Astrophys. J. 373, 277-284, DOI: $10.1086 / 170048$ (1991).

2. Fenimore, E. E., Epstein, R. I. \& Ho, C. The escape of $100 \mathrm{MeV}$ photons from cosmological gamma-ray bursts. @GRAVEACCENT@aaps 97, 59-62 (1993).

3. Woods, E. \& Loeb, A. Empirical Constraints on Source Properties and Host Galaxies of Cosmological Gamma-Ray Bursts. Astrophys. J. 453, 583, DOI: 10.1086/176421 (1995). astro-ph/9503070.

4. Lithwick, Y. \& Sari, R. Lower Limits on Lorentz Factors in Gamma-Ray Bursts. Astrophys. J. 555, 540-545, DOI: 10.1086/321455 (2001). astro-ph/0011508.

5. Zhao, X.-H., Li, Z. \& Bai, J.-M. The Bulk Lorentz Factors of Fermi-LAT Gamma Ray Bursts. Astrophys. J. 726, 89, DOI: 10.1088/0004-637X/726/2/89 (2011). 1005.5229.

6. Zou, Y.-C., Fan, Y.-Z. \& Piran, T. A Revised Limit of the Lorentz Factors of Gamma-ray Bursts with Two Emitting Regions. Astrophys. 726, L2, DOI: 10.1088/2041-8205/726/1/L2 (2011). 1008.2253.

7. Racusin, J. L. et al. Fermi and Swift Gamma-ray Burst Afterglow Population Studies. Astrophys. J. 738, 138, DOI: 10.1088/0004-637X/738/2/138 (2011). 1106.2469.

8. Nousek, J. A. et al. Evidence for a Canonical Gamma-Ray Burst Afterglow Light Curve in the Swift XRT Data. Astrophys. J. 642, 389-400, DOI: 10.1086/500724 (2006). astro-ph/0508332.

9. O'Brien, P. T. et al. The Early X-Ray Emission from GRBs. Astrophys. J. 647, 1213-1237, DOI: 10.1086/505457 (2006). astro-ph/0601125.

10. Zhang, B. et al. Physical Processes Shaping Gamma-Ray Burst X-Ray Afterglow Light Curves: Theoretical Implications from the Swift X-Ray Telescope Observations. Astrophys. J. 642, 354-370, DOI: $10.1086 / 500723$ (2006). astro-ph/0508321.

11. Liang, E.-W., Zhang, B.-B. \& Zhang, B. A Comprehensive Analysis of Swift XRT Data. II. Diverse Physical Origins of the Shallow Decay Segment. Astrophys. J. 670, 565-583, DOI: 10.1086/521870 (2007). 0705.1373. 
12. Srinivasaragavan, G. P. et al. On the Investigation of the Closure Relations for Gamma-Ray Bursts Observed by Swift in the Post-plateau Phase and the GRB Fundamental Plane. Astrophys. J. 903, 18, DOI: 10.3847/1538-4357/abb702 (2020). 2009.06740.

13. Meszaros, P. \& Rees, M. J. Relativistic fireballs and their impact on external matter - Models for cosmological gamma-ray bursts. Astrophys. J. 405, 278-284, DOI: 10.1086/172360 (1993).

14. Chevalier, R. A. \& Li, Z.-Y. Gamma-Ray Burst Environments and Progenitors. Astrophys. 520, L29-L32, DOI: 10.1086/312147 (1999). astro-ph/9904417.

15. Blandford, R. D. \& McKee, C. F. Fluid dynamics of relativistic blast waves. Phys. Fluids 19, 1130-1138, DOI: 10.1063/1.861619 (1976).

16. Ghisellini, G., Padovani, P., Celotti, A. \& Maraschi, L. Relativistic Bulk Motion in Active Galactic Nuclei. Astrophys. J. 407, 65, DOI: 10.1086/172493 (1993).

17. Evans, P. A. et al. Methods and results of an automatic analysis of a complete sample of Swift-XRT observations of GRBs. Mon. Not. R. Astron. Soc. 397, 1177-1201, DOI: 10.1111/j.1365-2966.2009. 14913.x (2009). 0812.3662.

18. Gehrels, N. et al. The Swift Gamma-Ray Burst Mission. Astrophys. J. 611, 1005-1020, DOI: 10.1086/422091 (2004). astro-ph/0405233.

19. Dainotti, M. G. et al. The Optical Luminosity-Time Correlation for More than 100 Gamma-Ray Burst Afterglows. Astrophys. 905, L26, DOI: 10.3847/2041-8213/abcda9 (2020). 2011.14493.

20. Sari, R., Piran, T. \& Narayan, R. Spectra and Light Curves of Gamma-Ray Burst Afterglows. Astrophys. 497, L17+, DOI: 10.1086/311269 (1998). arXiv:astro-ph/9712005.

21. Racusin, J. L. et al. Jet Breaks and Energetics of Swift Gamma-Ray Burst X-Ray Afterglows. Astrophys. J. 698, 43-74, DOI: 10.1088/0004-637X/698/1/43 (2009). 0812.4780.

22. Zhang, B., Wang, Y. \& Li, L. Dissecting the Energy Budget of a Gamma-Ray Burst Fireball. Astrophys. 909, L3, DOI: 10.3847/2041-8213/abe6ab (2021). 2102.04968.

23. Dainotti, M. G. et al. On the Existence of the Plateau Emission in High-energy Gamma-Ray Burst Light Curves Observed by Fermi-LAT. Astrophys. J. S. 255, 13, DOI: 10.3847/1538-4365/abfe17 (2021). 2105.07357.

24. Ajello, M. et al. A Decade of Gamma-Ray Bursts Observed by Fermi-LAT: The Second GRB Catalog. Astrophys. J. 878, 52, DOI: 10.3847/1538-4357/ab1d4e (2019). 1906.11403.

25. Yamazaki, R., Sato, Y., Sakamoto, T. \& Serino, M. Less noticeable shallow decay phase in early $\mathrm{X}$-ray afterglows of GeV/TeV-detected gamma-ray bursts. Mon. Not. R. Astron. Soc. 494, 5259-5269, DOI: 10.1093/mnras/staa1095 (2020). 1910.04097.

26. Meszaros, P. \& Rees, M. J. Optical and Long-Wavelength Afterglow from Gamma-Ray Bursts. Astrophys. J. 476, 232-+, DOI: 10.1086/303625 (1997). arXiv:astro-ph/9606043.

27. Kobayashi, S., Piran, T. \& Sari, R. Can Internal Shocks Produce the Variability in Gamma-Ray Bursts? Astrophys. J. 490, 92, DOI: 10.1086/512791 (1997). astro-ph/9705013.

28. Panaitescu, A. \& Kumar, P. Properties of Relativistic Jets in Gamma-Ray Burst Afterglows. Astrophys. J. 571, 779-789, DOI: 10.1086/340094 (2002).

29. Zhang, B., Kobayashi, S. \& Mészáros, P. Gamma-Ray Burst Early Optical Afterglows: Implications for the Initial Lorentz Factor and the Central Engine. Astrophys. J. 595, 950-954, DOI: 10.1086/ 377363 (2003). astro-ph/0302525. 
30. Molinari, E. et al. REM observations of GRB 060418 and GRB 060607A: the onset of the afterglow and the initial fireball Lorentz factor determination. Astron. Astrophys. 469, L13-L16, DOI: 10. 1051/0004-6361:20077388 (2007). astro-ph/0612607.

31. Rykoff, E. S. et al. Looking Into the Fireball: ROTSE-III and Swift Observations of Early Gammaray Burst Afterglows. Astrophys. J. 702, 489-505, DOI: 10.1088/0004-637X/702/1/489 (2009). 0904.0261.

32. Liang, E.-W. et al. Constraining Gamma-ray Burst Initial Lorentz Factor with the Afterglow Onset Feature and Discovery of a Tight $\Gamma_{0}-\mathrm{E}_{\text {gamma,iso }}$ Correlation. Astrophys. J. 725, 2209-2224, DOI: 10.1088/0004-637X/725/2/2209 (2010). 0912.4800.

33. Burrows, D. N. et al. Bright X-ray Flares in Gamma-Ray Burst Afterglows. Sci. 309, 1833-1835, DOI: 10.1126/science.1116168 (2005). astro-ph/0506130.

34. Chincarini, G. et al. The First Survey of X-Ray Flares from Gamma-Ray Bursts Observed by Swift: Temporal Properties and Morphology. Astrophys. J. 671, 1903-1920, DOI: 10.1086/521591 (2007). astro-ph/0702371.

35. Falcone, A. D. et al. The First Survey of X-Ray Flares from Gamma-Ray Bursts Observed by Swift: Spectral Properties and Energetics. Astrophys. J. 671, 1921-1938, DOI: 10.1086/523296 (2007). 0706.1564.

36. Chincarini, G. et al. Unveiling the origin of X-ray flares in gamma-ray bursts. Mon. Not. R. Astron. Soc. 406, 2113-2148, DOI: 10.1111/j.1365-2966.2010.17037.x (2010). 1004.0901.

37. Margutti, R. et al. On the average gamma-ray burst X-ray flaring activity. Mon. Not. R. Astron. Soc. 410, 1064-1075, DOI: 10.1111/j.1365-2966.2010.17504.x (2011). 1009.0172.

38. Pe'er, A., Ryde, F., Wijers, R. A. M. J., Mészáros, P. \& Rees, M. J. A New Method of Determining the Initial Size and Lorentz Factor of Gamma-Ray Burst Fireballs Using a Thermal Emission Component. Astrophys. 664, L1-L4, DOI: 10.1086/520534 (2007). arXiv:astro-ph/0703734.

39. Yu, H.-F., Dereli-Bégué, H. \& Ryde, F. Bayesian Time-resolved Spectroscopy of GRB Pulses. Astrophys. J. 886, 20, DOI: 10.3847/1538-4357/ab488a (2019). 1810.07313.

40. Acuner, Z., Ryde, F., Pe'er, A., Mortlock, D. \& Ahlgren, B. The Fraction of Gamma-Ray Bursts with an Observed Photospheric Emission Episode. Astrophys. J. 893, 128, DOI: 10.3847/1538-4357/ ab80c7 (2020). 2003.06223.

41. Dereli-Bégué, H., Pe'er, A. \& Ryde, F. Classification of Photospheric Emission in Short GRBs. Astrophys. J. 897, 145, DOI: 10.3847/1538-4357/ab9a2d (2020). 2002.06408.

42. Chevalier, R. A., Li, Z.-Y. \& Fransson, C. The Diversity of Gamma-Ray Burst Afterglows and the Surroundings of Massive Stars. Astrophys. J. 606, 369-380, DOI: 10.1086/382867 (2004). astro-ph/0311326.

43. Eldridge, J. J., Genet, F., Daigne, F. \& Mochkovitch, R. The circumstellar environment of WolfRayet stars and gamma-ray burst afterglows. Mon. Not. R. Astron. Soc. 367, 186-200, DOI: 10.1111/j.1365-2966.2005.09938.x (2006). astro-ph/0509749.

44. Granot, J. \& Kumar, P. Distribution of gamma-ray burst ejecta energy with Lorentz factor. Mon. Not. R. Astron. Soc. 366, L13-L16, DOI: 10.1111/j.1745-3933.2005.00121.x (2006). astro-ph/0511049. 
45. Fan, Y. \& Piran, T. Gamma-ray burst efficiency and possible physical processes shaping the early afterglow. Mon. Not. R. Astron. Soc. 369, 197-206, DOI: 10.1111/j.1365-2966.2006.10280.x (2006). astro-ph/0601054.

46. Panaitescu, A., Mészáros, P., Gehrels, N., Burrows, D. \& Nousek, J. Analysis of the X-ray emission of nine Swift afterglows. Mon. Not. R. Astron. Soc. 366, 1357-1366, DOI: 10.1111/j.1365-2966. 2005.09900.x (2006). astro-ph/0508340.

47. Ghisellini, G., Ghirlanda, G., Nava, L. \& Firmani, C. "Late Prompt" Emission in Gamma-Ray Bursts? Astrophys. 658, L75-L78, DOI: 10.1086/515570 (2007). astro-ph/0701430.

48. Metzger, B. D., Giannios, D., Thompson, T. A., Bucciantini, N. \& Quataert, E. The protomagnetar model for gamma-ray bursts. Mon. Not. R. Astron. Soc. 413, 2031-2056, DOI: 10.1111/j.1365-2966. 2011.18280.x (2011). 1012.0001.

49. Rowlinson, A. et al. Constraining properties of GRB magnetar central engines using the observed plateau luminosity and duration correlation. Mon. Notices Royal Astron. Soc. 443, 1779-1787, DOI: 10.1093/mnras/stu1277 (2014). https://academic.oup.com/mnras/article-pdf/443/2/1779/3703047/ stu1277.pdf.

50. Rea, N. et al. Constraining the GRB-magnetar model by means of the Galactic pulsar population. The Astrophys. J. 813, 92, DOI: 10.1088/0004-637x/813/2/92 (2015).

51. Stratta, G., Dainotti, M. G., Dall'Osso, S., Hernandez, X. \& De Cesare, G. On the Magnetar Origin of the GRBs Presenting X-Ray Afterglow Plateaus. Astrophys. J. 869, 155, DOI: 10.3847/1538-4357/ aadd8f (2018). 1804.08652.

52. Matsumoto, T., Kimura, S. S., Murase, K. \& Mészáros, P. Linking extended and plateau emissions of short gamma-ray bursts. Mon. Not. R. Astron. Soc. 493, 783-791, DOI: 10.1093/mnras/staa305 (2020). 2001.09851.

53. Zhao, L. et al. The Second Plateau in X-Ray Afterglow Providing Additional Evidence for Rapidly Spinning Magnetars as the GRB Central Engine. Astrophys. J. 896, 42, DOI: 10.3847/1538-4357/ ab8f91 (2020). 2005.00768.

54. Çıkıntoğlu, S., Şaşmaz Muş, S. \& Ekşi, K. Y. The initial evolution of millisecond magnetars: an analytical solution. Mon. Not. R. Astron. Soc. 496, 2183-2190, DOI: 10.1093/mnras/staa1556 (2020). 1910.00554 .

55. Berger, E. et al. A common origin for cosmic explosions inferred from calorimetry of GRB030329. Nat. 426, 154-157, DOI: 10.1038/nature01998 (2003). astro-ph/0308187.

56. Huang, Y. F., Wu, X. F., Dai, Z. G., Ma, H. T. \& Lu, T. Rebrightening of XRF 030723: Further Evidence for a Two-Component Jet in a Gamma-Ray Burst. Astrophys. J. 605, 300-306, DOI: 10.1086/382202 (2004). astro-ph/0309360.

57. Granot, J., Königl, A. \& Piran, T. Implications of the early X-ray afterglow light curves of Swift gamma-ray bursts. Mon. Not. R. Astron. Soc. 370, 1946-1960, DOI: 10.1111/j.1365-2966.2006. 10621.x (2006). astro-ph/0601056.

58. Racusin, J. L. et al. Broadband observations of the naked-eye $\gamma$-ray burst GRB080319B. Nat. 455, 183-188, DOI: 10.1038/nature07270 (2008). 0805.1557.

59. Toma, K., Ioka, K., Yamazaki, R. \& Nakamura, T. Shallow Decay of Early X-Ray Afterglows from Inhomogeneous Gamma-Ray Burst Jets. Astrophys. 640, L139-L142, DOI: 10.1086/503384 (2006). astro-ph/0511718. 
60. Ioka, K., Toma, K., Yamazaki, R. \& Nakamura, T. Efficiency crisis of swift gamma-ray bursts with shallow X-ray afterglows: prior activity or time-dependent microphysics? Astron. Astrophys. 458, 7-12, DOI: 10.1051/0004-6361:20064939 (2006). astro-ph/0511749.

61. Shao, L. \& Dai, Z. G. Behavior of X-Ray Dust Scattering and Implications for X-Ray Afterglows of Gamma-Ray Bursts. Astrophys. J. 660, 1319-1325, DOI: 10.1086/513139 (2007). astro-ph/0703009.

62. Uhm, Z. L. \& Beloborodov, A. M. On the Mechanism of Gamma-Ray Burst Afterglows. Astrophys. 665, L93-L96, DOI: 10.1086/519837 (2007). astro-ph/0701205.

63. Genet, F., Daigne, F. \& Mochkovitch, R. Can the early X-ray afterglow of gamma-ray bursts be explained by a contribution from the reverse shock? Mon. Not. R. Astron. Soc. 381, 732-740, DOI: 10.1111/j.1365-2966.2007.12243.x (2007). astro-ph/0701204.

64. Hascoët, R., Beloborodov, A. M., Daigne, F. \& Mochkovitch, R. Estimates for Lorentz Factors of Gamma-Ray Bursts from Early Optical Afterglow Observations. Astrophys. J. 782, 5, DOI: 10.1088/0004-637X/782/1/5 (2014). 1304.5813.

65. Lyutikov, M. \& Camilo Jaramillo, J. Early GRB Afterglows from Reverse Shocks in Ultra-relativistic, Long-lasting Winds. Astrophys. J. 835, 206, DOI: 10.3847/1538-4357/835/2/206 (2017). 1612. 01162.

66. Barkov, M. V., Luo, Y. \& Lyutikov, M. Dynamics and Emission of Wind-powered Afterglows of Gamma-Ray Bursts: Flares, Plateaus, and Steep Decays. Astrophys. J. 907, 109, DOI: 10.3847/ 1538-4357/abd5c2 (2021). 2004.13600.

67. Eichler, D. \& Granot, J. The Case for Anisotropic Afterglow Efficiency within Gamma-Ray Burst Jets. Astrophys. 641, L5-L8, DOI: 10.1086/503667 (2006). astro-ph/0509857.

68. Eichler, D. \& Manis, H. Spectral Lags Explained as Scattering from Accelerated Scatterers. Astrophys. 689, L85, DOI: 10.1086/595839 (2008). 0810.3006.

69. Eichler, D. Cloaked Gamma-Ray Bursts. Astrophys. 787, L32, DOI: 10.1088/2041-8205/787/2/L32 (2014). 1402.0245.

70. Oganesyan, G. et al. Structured Jets and X-Ray Plateaus in Gamma-Ray Burst Phenomena. Astrophys. J. 893, 88, DOI: 10.3847/1538-4357/ab8221 (2020). 1904.08786.

71. Beniamini, P., Duque, R., Daigne, F. \& Mochkovitch, R. X-ray plateaus in gamma-ray bursts' light curves from jets viewed slightly off-axis. Mon. Not. R. Astron. Soc. 492, 2847-2857, DOI: 10.1093/mnras/staa070 (2020). 1907.05899.

72. Kumar, P. \& Zhang, B. The physics of gamma-ray bursts and relativistic jets. Phys. Reports 561, 1-109, DOI: 10.1016/j.physrep.2014.09.008 (2015). 1410.0679.

73. Shen, R. \& Matzner, C. D. Coasting External Shock in Wind Medium: An Origin for the X-Ray Plateau Decay Component in Swift Gamma-Ray Burst Afterglows. Astrophys. J. 744, 36, DOI: 10.1088/0004-637X/744/1/36 (2012). 1109.3453.

74. Dainotti, M. G. et al. The X-Ray Fundamental Plane of the Platinum Sample, the Kilonovae, and the SNe Ib/c Associated with GRBs. The Astrophys. J. 904, 97, DOI: 10.3847/1538-4357/abbe8a (2020).

75. Dainotti, M. G. et al. Closure relations during the plateau emission of Swift GRBs and the fundamental plane. Publ. Astron. Soc. Jpn. 73, 970-1000, DOI: 10.1093/pasj/psab057 (2021). 
76. Dainotti, M. G. et al. On the Existence of the Plateau Emission in High-energy Gamma-Ray Burst Light Curves Observed by Fermi-LAT. The Astrophys. J. Suppl. Ser. 255, 13, DOI: 10.3847/ 1538-4365/abfe17 (2021).

77. Dainotti, M. G., Postnikov, S., Hernandez, X. \& Ostrowski, M. A Fundamental Plane for Long Gamma-Ray Bursts with X-Ray Plateaus. The Astrophys. J. 825, L20, DOI: 10.3847/2041-8205/ $825 / 2 / 120(2016)$.

78. Dainotti, M. G. et al. A Study of the Gamma-Ray Burst Fundamental Plane. The Astrophys. J. 848, 88, DOI: 10.3847/1538-4357/aa8a6b (2017).

79. Willingale, R. et al. Testing the Standard Fireball Model of Gamma-Ray Bursts Using Late XRay Afterglows Measured by Swift. Astrophys. J. 662, 1093-1110, DOI: 10.1086/517989 (2007). astro-ph/0612031.

80. Ioka, K., Kobayashi, S. \& Zhang, B. Variabilities of Gamma-Ray Burst Afterglows: Long-acting Engine, Anisotropic Jet, or Many Fluctuating Regions? Astrophys. J. 631, 429-434, DOI: 10.1086/ 432567 (2005). astro-ph/0409376.

81. Boër, M. \& Gendre, B. Evidences for two Gamma-Ray Burst afterglow emission regimes. Astron. Astrophys. 361, L21-L24 (2000). astro-ph/0008385.

82. Gendre, B., Galli, A. \& Boër, M. X-Ray Afterglow Light Curves: Toward A Standard Candle? Astrophys. J. 683, 620-629, DOI: 10.1086/589805 (2008).

83. Oates, S. R. et al. A correlation between the intrinsic brightness and average decay rate of Swift/UVOT gamma-ray burst optical/ultraviolet light curves. Mon. Not. R. Astron. Soc. 426, L86-L90, DOI: 10.1111/j.1745-3933.2012.01331.x (2012). 1208.1856.

84. Racusin, J. L., Oates, S. R., de Pasquale, M. \& Kocevski, D. A Correlation between the Intrinsic Brightness and Average Decay Rate of Gamma-Ray Burst X-Ray Afterglow Light Curves. Astrophys. J. 826, 45, DOI: 10.3847/0004-637X/826/1/45 (2016). 1605.00719.

85. Dereli, H. et al. A Study of GRBs with Low-luminosity Afterglows. Astrophys. J. 850, 117, DOI: 10.3847/1538-4357/aa947d (2017).

86. Lazzati, D. \& Perna, R. X-ray flares and the duration of engine activity in gamma-ray bursts. Mon. Not. R. Astron. Soc. 375, L46-L50, DOI: 10.1111/j.1745-3933.2006.00273.x (2007). astro-ph/ 0610730 .

87. Evans, P. A. et al. An online repository of Swift/XRT light curves of $\gamma$-ray bursts. Astron. Astrophys. 469, 379-385, DOI: 10.1051/0004-6361:20077530 (2007). 0704.0128.

88. Goodman, J. \& Weare, J. Ensemble samplers with affine invariance. Commun. Appl. Math. Comput. Sci. 5, 65-80, DOI: 10.2140/camcos.2010.5.65 (2010).

89. Zaninoni, E., Bernardini, M. G., Margutti, R., Oates, S. \& Chincarini, G. Gamma-ray burst optical light-curve zoo: comparison with X-ray observations. Astron. Astrophys. 557, A12, DOI: 10.1051/0004-6361/201321221 (2013). 1303.6924.

90. De Pasquale, M. et al. The central engine of GRB 130831A and the energy breakdown of a relativistic explosion. Mon. Not. R. Astron. Soc. 455, 1027-1042, DOI: 10.1093/mnras/stv2280 (2016). 1509.09234.

91. Filgas, R. et al. GRB 091029: at the limit of the fireball scenario. Astron. Astrophys. 546, A101, DOI: 10.1051/0004-6361/201219583 (2012). 1209.4658. 
92. Cucchiara, A. et al. Constraining Gamma-Ray Burst Emission Physics with Extensive Earlytime, Multiband Follow-up. Astrophys. J. 743, 154, DOI: 10.1088/0004-637X/743/2/154 (2011). 1107.3352 .

93. de Pasquale, M. et al. VizieR Online Data Catalog: Photometry of the afterglow of GRB 130831A (De Pasquale+, 2016). VizieR Online Data Catalog J/MNRAS/455/1027 (2018).

94. Izzo, L. et al. Signatures of a jet cocoon in early spectra of a supernova associated with a $\gamma$-ray burst. Nat. 565, 324-327, DOI: 10.1038/s41586-018-0826-3 (2019). 1901.05500.

95. Oates, S. R. et al. A statistical study of gamma-ray burst afterglows measured by the Swift Ultraviolet Optical Telescope. Mon. Not. R. Astron. Soc. 395, 490-503, DOI: 10.1111/j.1365-2966.2009.14544.x (2009). 0901.3597.

96. Zafar, T. et al. The extinction curves of star-forming regions from $\mathrm{z}=0.1$ to 6.7 using GRB afterglow spectroscopy. Astron. Astrophys. 532, A143, DOI: 10.1051/0004-6361/201116663 (2011). 1102.1469.

97. Schlegel, D. J., Finkbeiner, D. P. \& Davis, M. Maps of Dust Infrared Emission for Use in Estimation of Reddening and Cosmic Microwave Background Radiation Foregrounds. Astrophys. J. 500, 525-553, DOI: 10.1086/305772 (1998). astro-ph/9710327.

98. Schady, P. et al. Dust and metal column densities in gamma-ray burst host galaxies. Mon. Not. R. Astron. Soc. 401, 2773-2792, DOI: 10.1111/j.1365-2966.2009.15861.x (2010). 0910.2590.

99. Pei, Y. C. Interstellar Dust from the Milky Way to the Magellanic Clouds. Astrophys. J. 395, 130, DOI: 10.1086/171637 (1992).

100. Tang, C.-H., Huang, Y.-F., Geng, J.-J. \& Zhang, Z.-B. Statistical Study of Gamma-Ray Bursts with a Plateau Phase in the X-Ray Afterglow. Astrophys. J. S. 245, 1, DOI: 10.3847/1538-4365/ab4711 (2019). 1905.07929.

101. Wright, E. L. A Cosmology Calculator for the World Wide Web. Publ. Astr. Soc. Pac. 118, 1711-1715, DOI: 10.1086/510102 (2006). astro-ph/0609593.

102. Dainotti, M. G., Ostrowski, M. \& Willingale, R. Towards a standard gamma-ray burst: tight correlations between the prompt and the afterglow plateau phase emission. Mon. Notices Royal Astron. Soc. 418, 2202-2206, DOI: 10.1111/j.1365-2966.2011.19433.x (2011). https://academic. oup.com/mnras/article-pdf/418/4/2202/18747557/mnras0418-2202.pdf.

103. Dainotti, M. et al. Luminosity-time and luminosity-luminosity correlations for GRB prompt and afterglow plateau emissions. Mon. Notices Royal Astron. Soc. 451, 3898-3908, DOI: 10.1093/mnras/ stv1229 (2015). https://academic.oup.com/mnras/article-pdf/451/4/3898/3889902/stv1229.pdf.

104. Dainotti, M. Gamma-ray Burst Correlations. 2053-2563 (IOP Publishing, 2019).

105. Chevalier, R. A. \& Li, Z.-Y. Wind Interaction Models for Gamma-Ray Burst Afterglows: The Case for Two Types of Progenitors. Astrophys. J. 536, 195-212, DOI: 10.1086/308914 (2000). astro-ph/9908272.

106. Pe'er, A. \& Wijers, R. A. M. J. The Signature of a Wind Reverse Shock in Gamma-Ray Burst Afterglows. Astrophys. J. 643, 1036-1046, DOI: $10.1086 / 500969$ (2006). arXiv:astro-ph/0511508.

107. Gao, H., Lei, W.-H., Zou, Y.-C., Wu, X.-F. \& Zhang, B. A complete reference of the analytical synchrotron external shock models of gamma-ray bursts. New Astron. Rev. 57, 141-190, DOI: 10.1016/j.newar.2013.10.001 (2013). 1310.2181. 
108. Bostanc1, Z. F., Kaneko, Y. \& Gögüus, E. Gamma-ray bursts with extended emission observed with BATSE. Mon. Not. R. Astron. Soc. 428, 1623-1630, DOI: 10.1093/mnras/sts157 (2013). 1210.2399.

109. Kaneko, Y., Bostanc1, Z. F., Göğüş, E. \& Lin, L. Short gamma-ray bursts with extended emission observed with Swift/BAT and Fermi/GBM. Mon. Not. R. Astron. Soc. 452, 824-837, DOI: 10.1093/ mnras/stv1286 (2015). 1506.05899.

110. Spitkovsky, A. On the Structure of Relativistic Collisionless Shocks in Electron-Ion Plasmas. Astrophys. 673, L39-L42, DOI: $10.1086 / 527374$ (2008). 0706.3126.

111. Wijers, R. A. M. J. \& Galama, T. J. Physical Parameters of GRB 970508 and GRB 971214 from Their Afterglow Synchrotron Emission. Astrophys. J. 523, 177-186, DOI: 10.1086/307705 (1999). astro-ph/9805341.

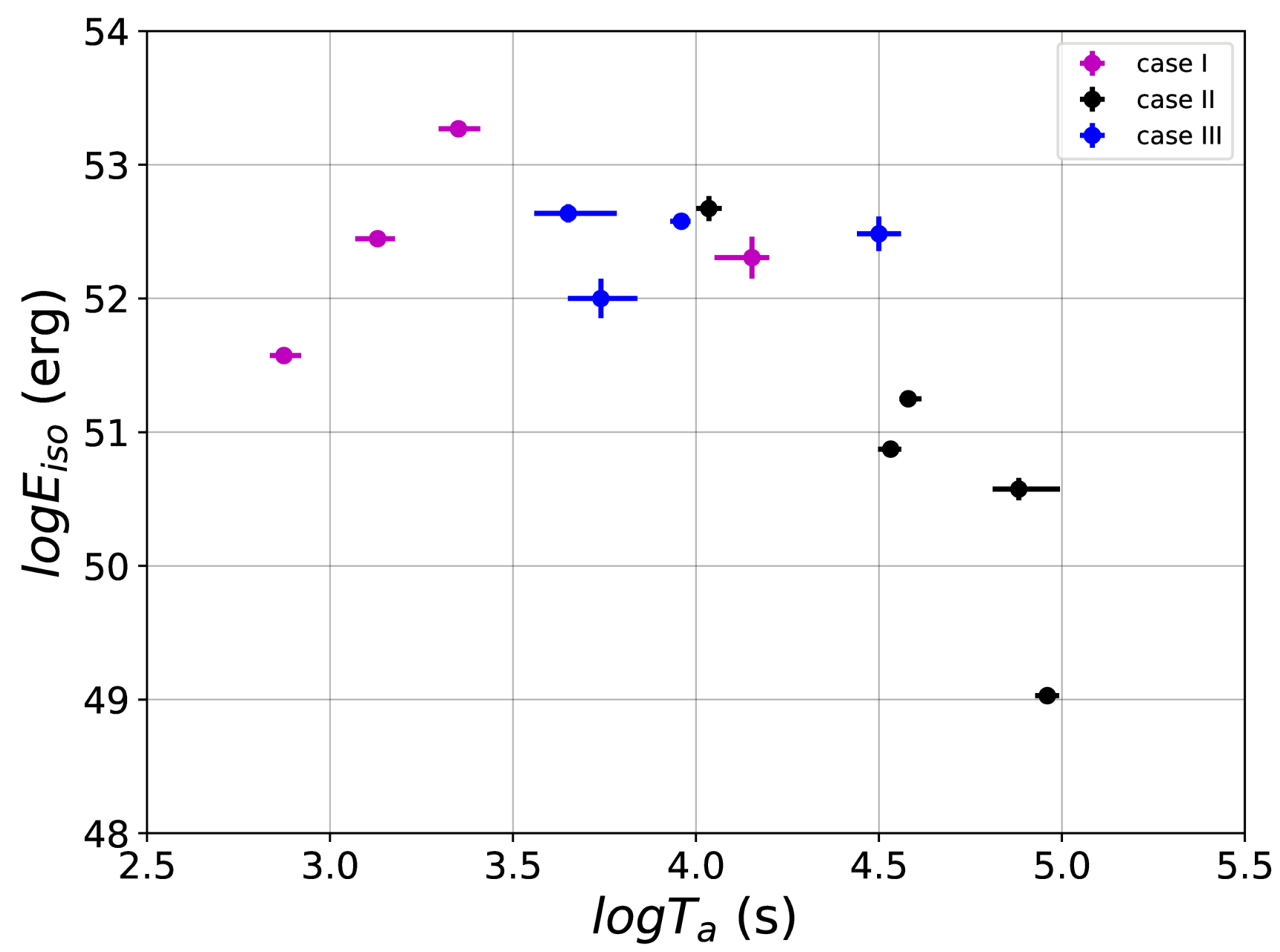

Figure 1. Isotropic Energy, $E_{i s o}$ as a function of time at the end of the plateau phase, $T_{a, X}$. Purple, black and blue points represent GRBs in the three different cases I, II, III respectively (see Tables 1, 2, 3). 


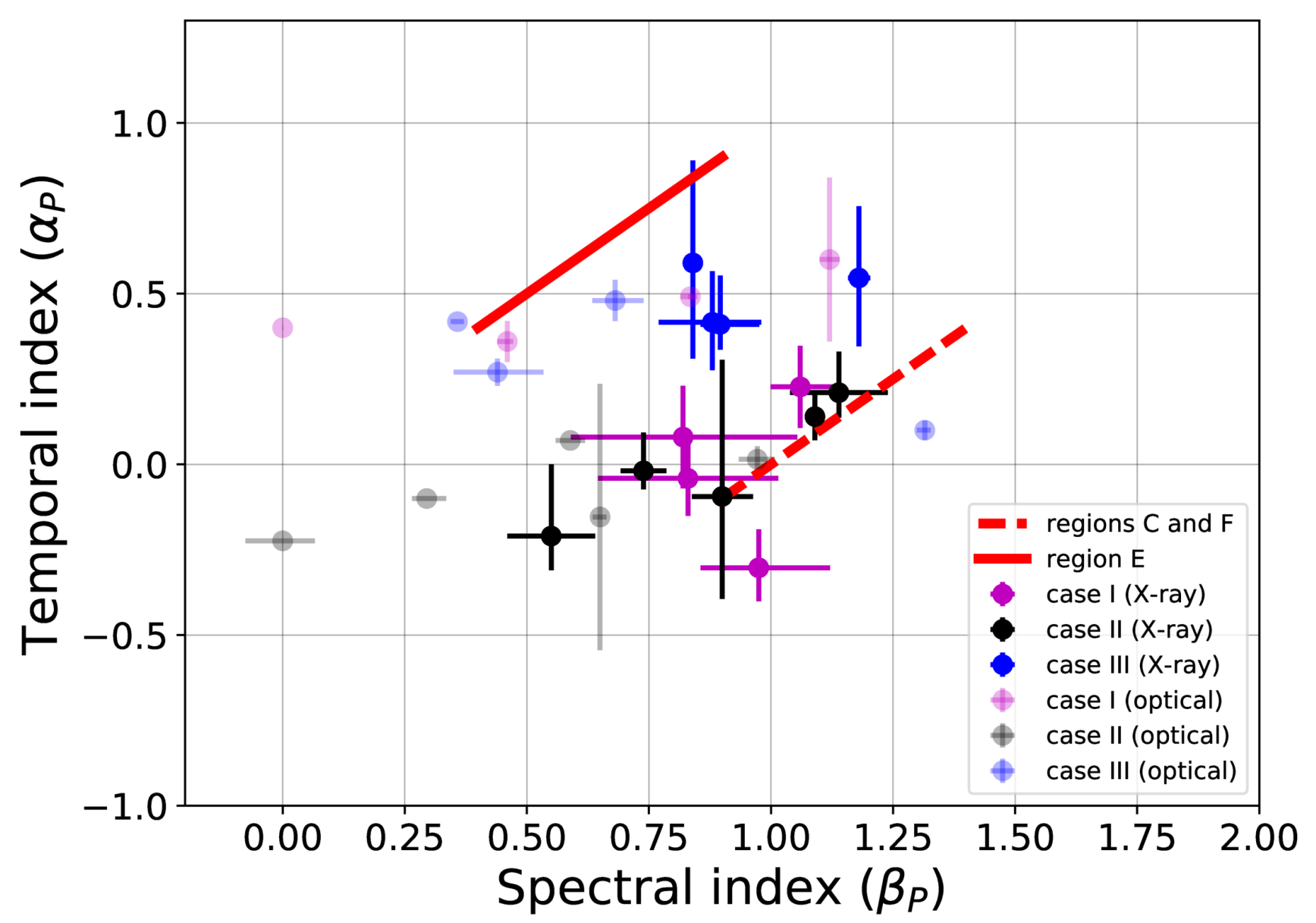

Figure 2. Temporal and spectral indices of the $13 \mathrm{GRBs}$ in our sample during the plateau phase. The purple, black, and blue data points are the slopes at the X-ray band, and the same color coding with lower transparency data points are the slopes obtained at the optical band of the GRBs in the three cases (I, II and III respectively). Red line show the closure relation obtained in region [E] in which $v_{m}<v_{\text {obs. }}<v_{c}$, and the dashed red line represents regions [C] and [F] in which $v_{c}<v_{m}<v_{\text {obs. }}$ or $v_{m}<v_{c}<v_{\text {obs. }}$. These lines are computed by using an electron power law index $p=1.8-2.8$. All the data points during the plateau phase both in X-ray and optical bands are consistent with the theoretical closure relations of the model. 


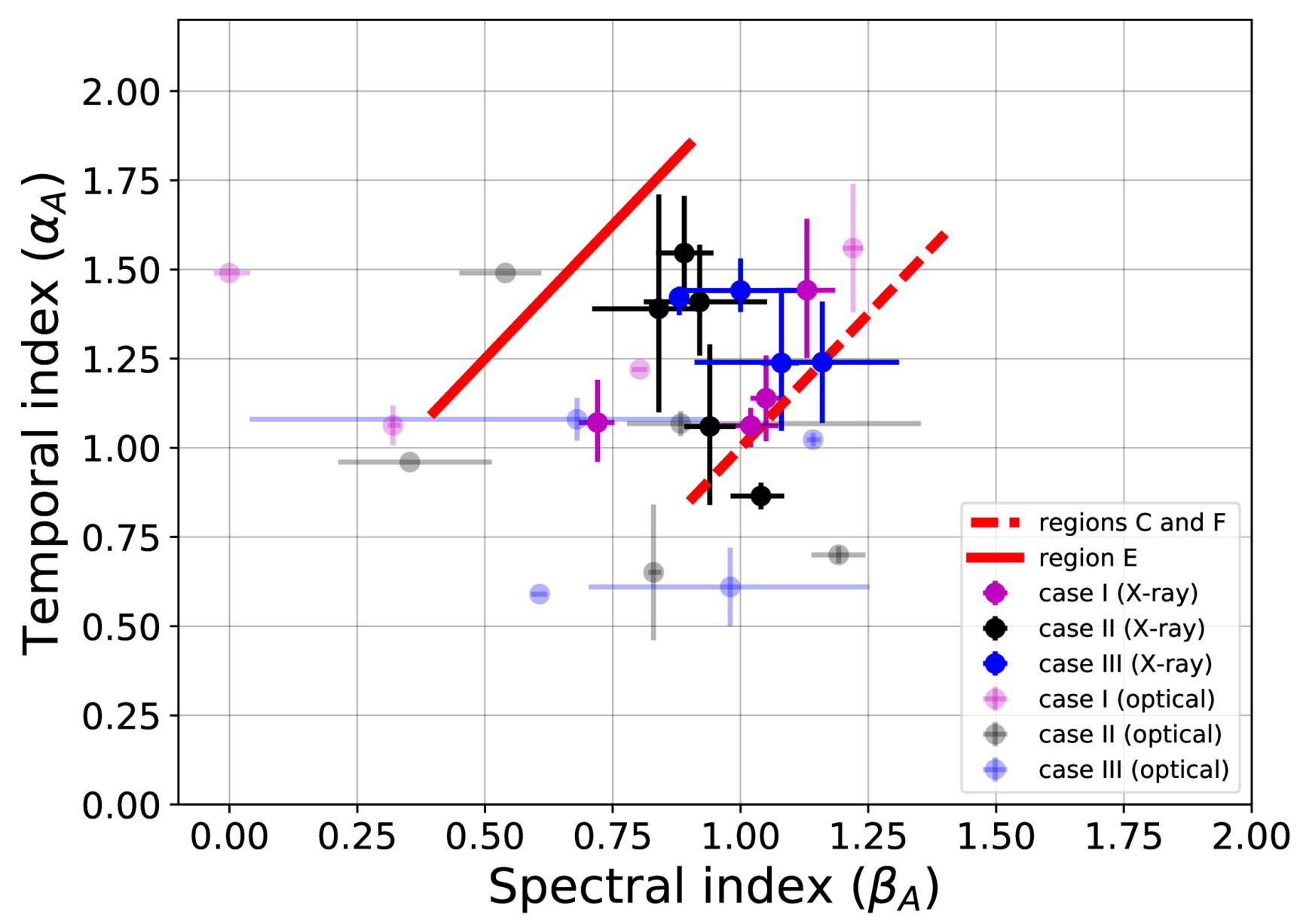

Figure 3. Temporal and spectral indices of 13 GRBs during the self-similar phase. Same color coding as in Figure 2 for the data points and regions. All the data points during the self-similar phase both in X-ray and optical bands are consistent with the theoretical closure relations of the model. 


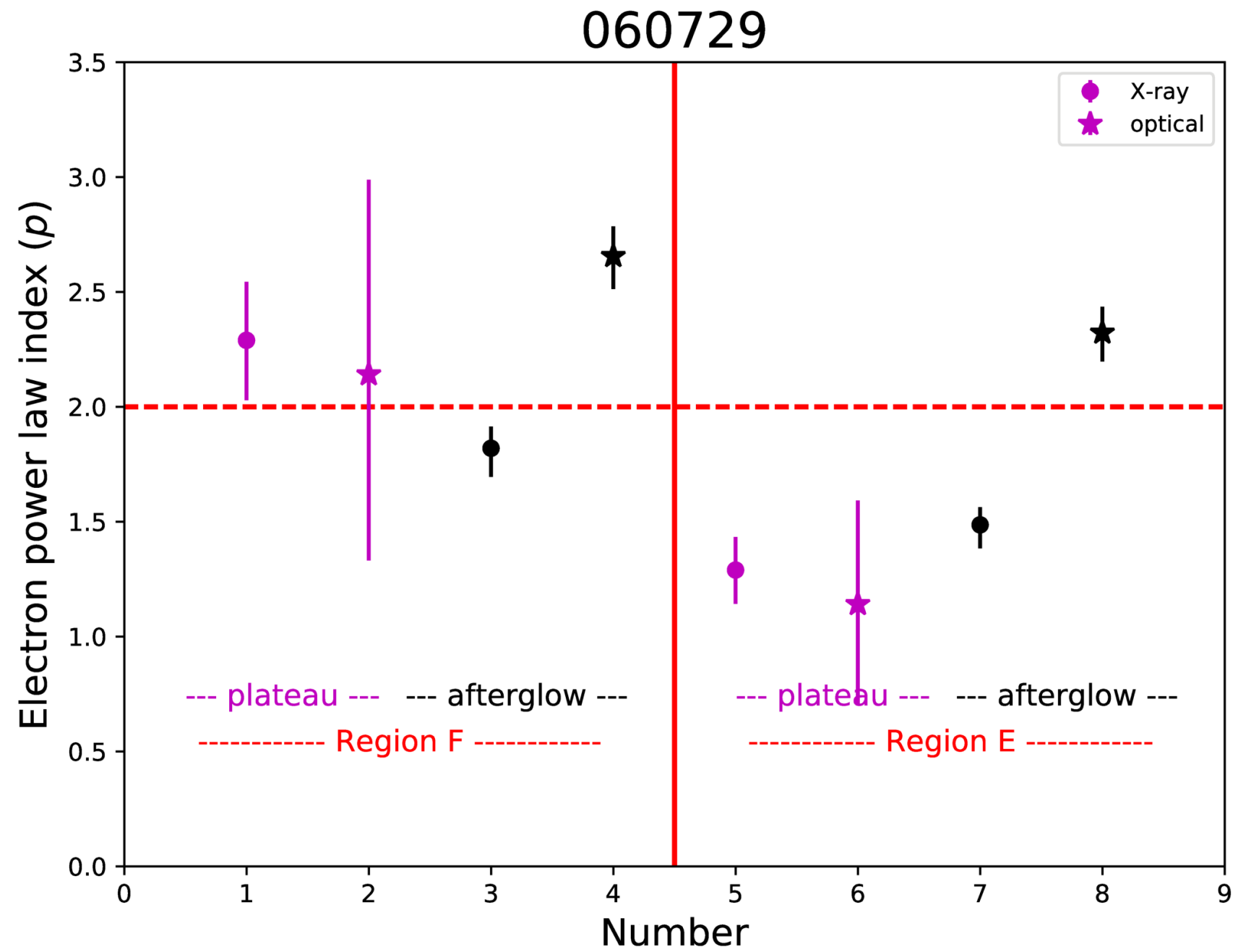

Figure 4. Extended Data: An example demonstrating how we determine the relevant region for a given source ([F] in this case) as well as the electron power law index $(p)$. Using the temporal slopes of both the X-ray (dot) and optical (star) light curves during both the plateau (purple) and self-similar (black) phases, we calculate the electron power law index under the assumption that the emission is in region $[\mathrm{F}]$ (left) and $[\mathrm{E}]$ (right). The independent calculations converge in region $[\mathrm{F}]$ except for one point while leading to different results under the assumption that the emission is in region [E]. We therefore conclude that the emission of GRB 060729 is in region [F]. The vertical red line separates the regions. Dashed line represents $p=2$. 


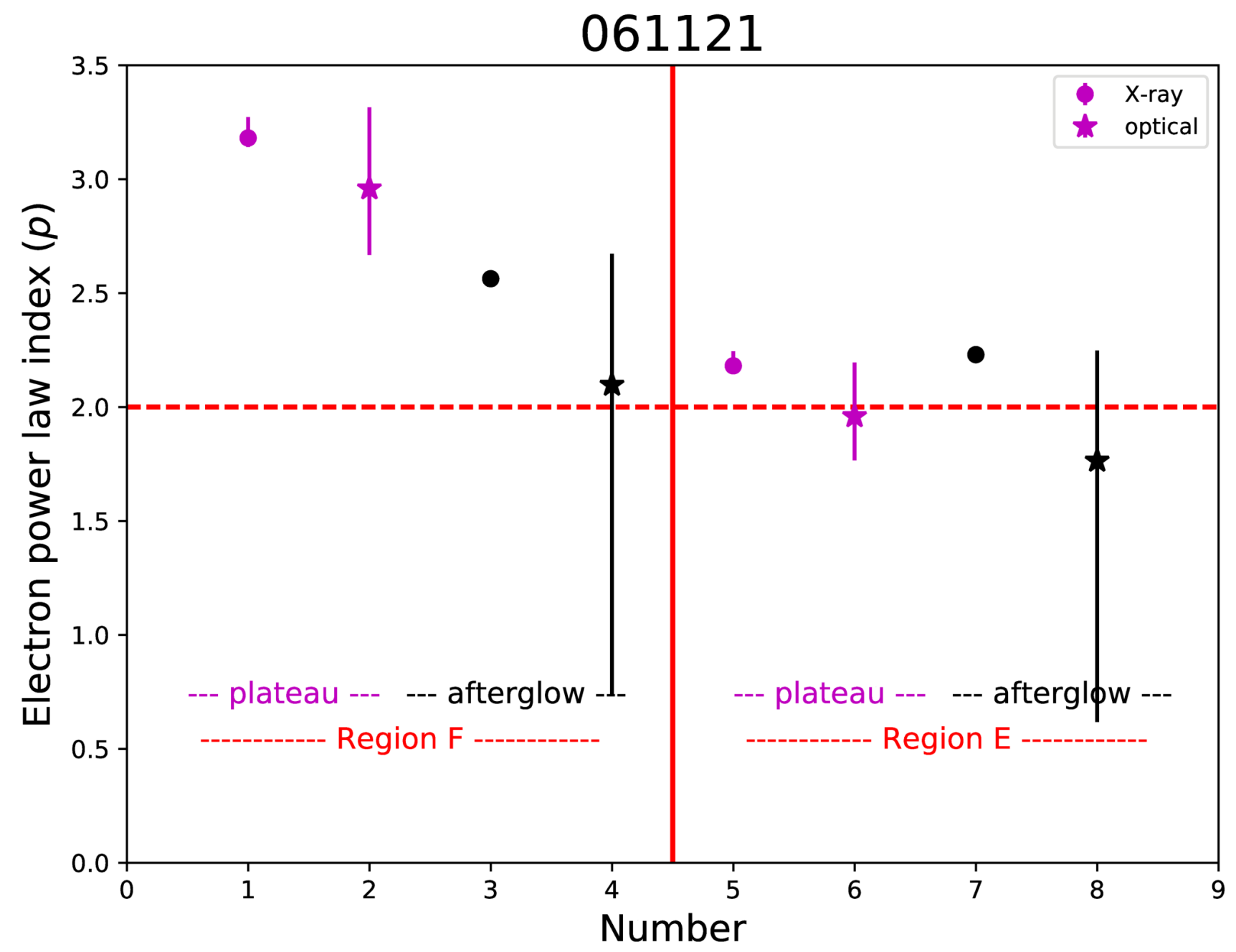

Figure 5. Extended Data: An example showing a burst which is in region [E]. Same explanation as in Figure 4. 


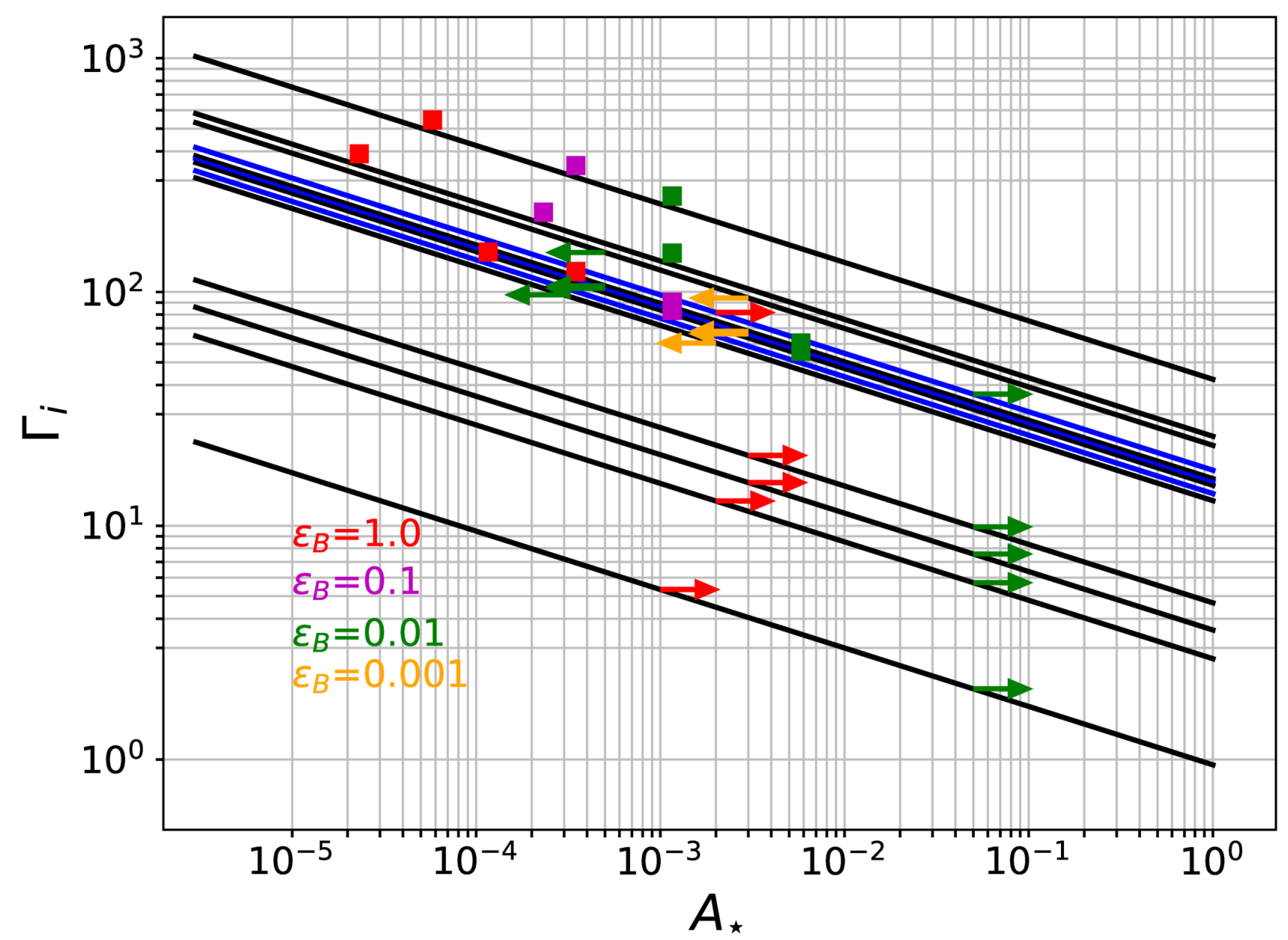

Figure 6. The initial GRB jet Lorentz factor, $\Gamma_{i}$ and the ambient density, $A_{\star}$ are marked by the black and blue (overlapped) lines, each corresponds to a different GRB. From top to bottom: GRBs 080607, 110213A, 060714, 080310, 061121, 060605, 130831A, 091029, 050319, 060729, 060614, 100418A, and 171205A. For a given $A_{\star}$, we determine the value of $\Gamma_{i}$ by using Equation 16 and knowing the burst energy and transition time. This gives the lines. In order to further constrain the values of these parameters, we assume knowledge of magnetization, $\varepsilon_{B}$, and use Equation 17 to deduce direct values of $A_{\star}$ and $\Gamma_{i}$ (squares) for cases I. For cases II and III, we use Equations 18 and 19 to compute the lower (upper) and upper (lower) limits of $A_{\star}\left(\Gamma_{i}\right)$ (arrows). In all cases, the constrain put by the magnetization $\left(\varepsilon_{B}\right)$ is inversely proportional to the ambient density. We consider $\varepsilon_{B}=1.0$ (equipartition), 0.1, 0.01 and 0.001 which are respectively presented by red, purple, green and orange colors. 


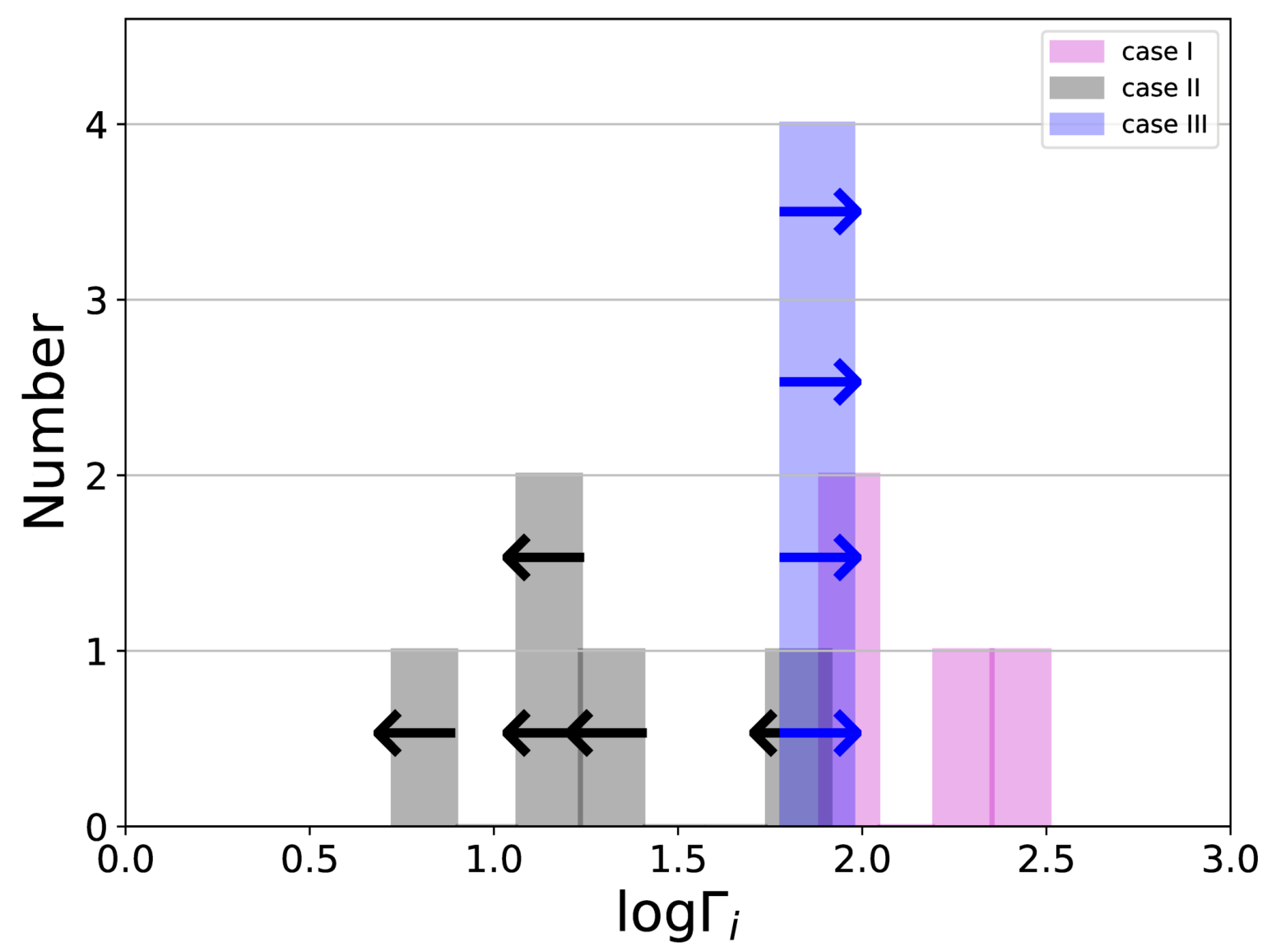

Figure 7. Histogram of the initial jet Lorentz factors of the $13 \mathrm{GRBs}$ in our sample. These values are obtained by assuming the fraction of energy in the magnetic field is $\varepsilon_{B}=0.1,1.0$ and 0.001 for the GRBs listed in different cases I, II, III respectively (see Tables 1,2,3). This choice is motivated to ensure that $\varepsilon_{e}$ is smaller than unity in all cases. Purple bars represent values deduced directly from the data (case I), the blue bars are lower limits (case II) and the black bars are upper limits (case III). Upper and lower limits are also marked by arrows. The average value of the initial GRB jet Lorentz factor is $\left\langle\Gamma_{i}\right\rangle \approx 85$, although the range span is between $5 \leq \Gamma_{i} \leq 322$ (see Table 7). We point out that GRB 080607 which has the highest value of $\Gamma_{i}$ has a large gap in its X-ray LC between the plateau and self-similar phases. Furthermore, GRB 171205A which has the lowest value of $\Gamma_{i}$ is associated with SN 2017iuk, therefore, both the optical plateau and self-similar slopes of this burst are effected by the SN bump (see further discussion in Method). 


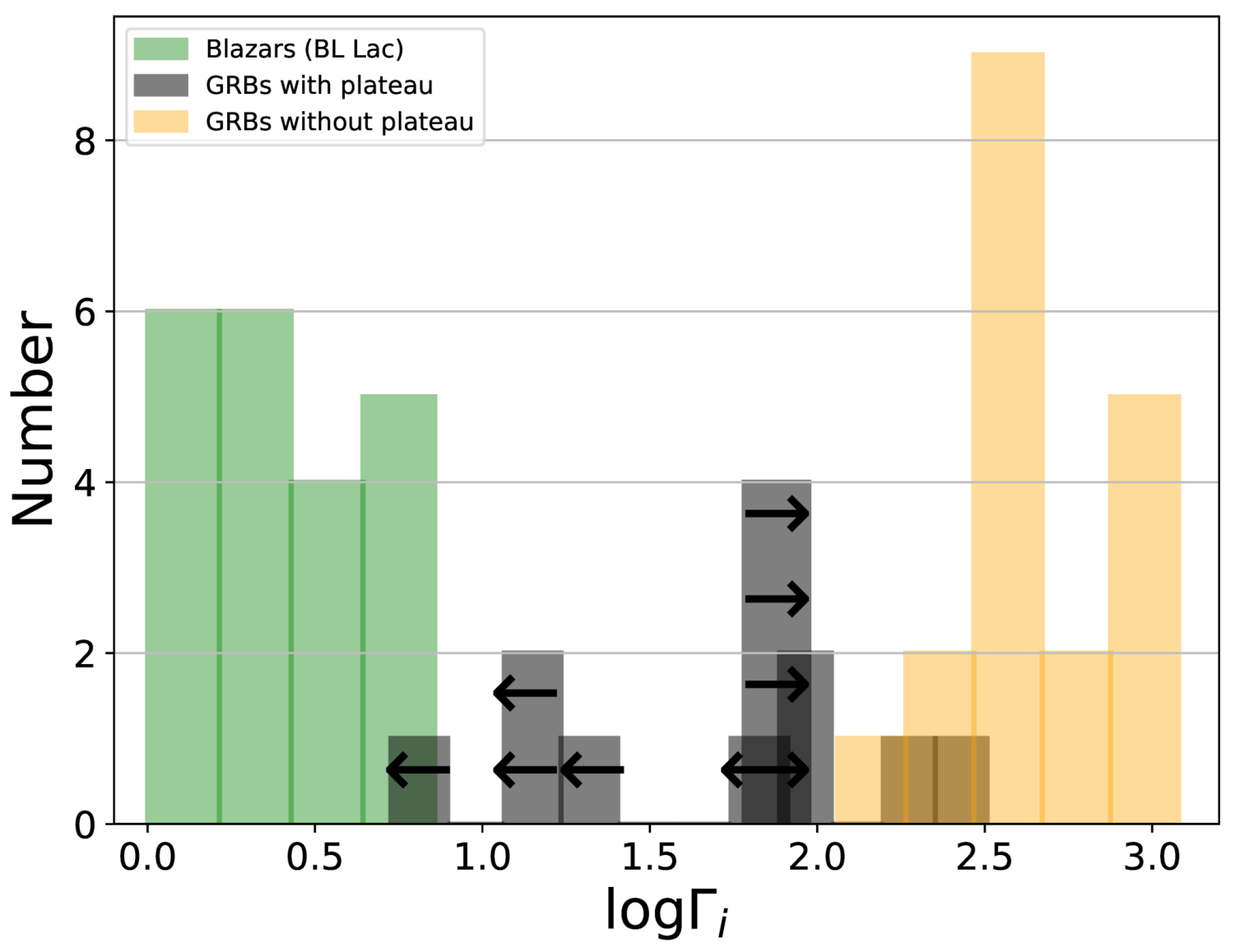

Figure 8. Histogram of the initial jet Lorentz factors. Black represents the sample of GRBs in our sample, as appear in Figure 7. Yellow represents GRBs observed by the Fermi-LAT instrument, taken from Ref. ${ }^{7}$, whose jet Lorentz factor is deduced using the opacity argument (GRBs 090926A, 090902B, 090510, 080916C; see Ref. ${ }^{7}$, Figure 11) and other GRBs that do not show a plateau phase (except 061021), taken from Ref. ${ }^{32}$ (table 6 there in). Green represents the inferred lower limit to the Lorentz factor of BL Lac objects (Blazars), taken from Ref. ${ }^{16}$ (see their table 2A). The values of the Lorentz factor we found, of few tens, fills the gap that previously existed in the range of initial jet velocities. 


\begin{tabular}{ccccc}
\hline GRB names & 080607 & 091029 & $110213 \mathrm{~A}$ & $130831 \mathrm{~A}$ \\
\hline $\log \mathrm{T}_{\mathrm{a}, \mathrm{X}}(\mathrm{s})$ & $3.35_{-0.05}^{+0.06}$ & $4.15_{-0.10}^{+0.05}$ & $3.13_{-0.06}^{+0.04}$ & $2.87_{-0.04}^{+0.05}$ \\
$\log \mathrm{T}_{\mathrm{b}, \mathrm{X}}(\mathrm{s})$ & $4.07_{-0.04}^{+0.05}$ & $\ldots$ & $3.97_{-0.03}^{+0.03}$ & $\ldots$ \\
$\alpha_{\mathrm{p}, \mathrm{X}}$ & $0.08_{-0.22}^{+0.23}$ & $-0.23_{-0.06}^{+0.09}$ & $0.30_{-0.11}^{+0.14}$ & $0.04_{-0.18}^{+0.19}$ \\
$\alpha_{\mathrm{A} 1, \mathrm{X}}$ & $-2.24_{-0.12}^{+0.11}$ & $-1.14_{-0.03}^{+0.03}$ & $-1.06_{-0.05}^{+0.05}$ & $-1.07_{-0.04}^{+0.03}$ \\
$\alpha_{\mathrm{A} 2, \mathrm{X}}$ & $-1.44_{-0.06}^{+0.06}$ & $\ldots$ & $-1.94_{-0.03}^{+0.04}$ & $\ldots$ \\
$\beta_{\mathrm{p}, \mathrm{X}}$ & $0.82_{-0.15}^{+0.15}$ & $1.06_{-0.12}^{+0.12}$ & $0.98_{-0.10}^{+0.11}$ & $0.83_{-0.11}^{+0.14}$ \\
$\beta_{\mathrm{A} 1, \mathrm{X}}$ & $1.20_{-0.15}^{+0.15}$ & $1.05_{-0.12}^{+0.12}$ & $1.02_{-0.05}^{+0.06}$ & $0.72_{-0.11}^{+0.12}$ \\
$\beta_{\mathrm{A} 2, \mathrm{X}}$ & $1.13_{-0.19}^{+0.20}$ & $\ldots$ & $1.14_{-0.19}^{+0.21}$ & $\ldots$ \\
$\log \mathrm{T}_{\mathrm{a}, \mathrm{O}}(\mathrm{s})$ & $3.40_{-0.02}^{+0.05}$ & $4.19_{-0.03}^{+0.03}$ & $3.34_{-0.02}^{+0.11}$ & $3.69_{-0.02}^{+0.01}$ \\
$\log \mathrm{T}_{\mathrm{b}, \mathrm{O}}(\mathrm{s})$ & $\ldots$ & $\ldots$ & $3.71_{-0.01}^{+0.13}$ & 931.0 \\
$\alpha_{\mathrm{p}, \mathrm{O}}$ & $-0.49_{-0.02}^{+0.01}$ & $-0.36_{-0.02}^{+0.01}$ & $-0.60_{-0.02}^{+0.02}$ & $-0.4_{-0.01}^{+0.01}$ \\
$\alpha_{\mathrm{A} 1, \mathrm{O}}$ & $-1.22_{-0.02}^{+0.01}$ & $-1.06_{-0.01}^{+0.01}$ & $0.68_{-0.07}^{+0.07}$ & $-1.49_{-0.03}^{+0.04}$ \\
$\alpha_{\mathrm{A} 2, \mathrm{O}}$ & $\ldots$ & $\ldots$ & $-1.56_{-0.02}^{+0.02}$ & $\ldots$ \\
$\beta_{\mathrm{p}, \mathrm{O}}$ & $-0.84_{-0.01}^{+0.01}$ & $-0.46_{-0.06}^{+0.06}$ & $\ldots$ & $\ldots$ \\
$\beta_{\mathrm{A} 1, \mathrm{O}}$ & $-0.80_{0.0}^{0.0}$ & $-0.32_{-0.06}^{+0.05}$ & $-1.12_{-0.24}^{+0.24}$ & $\ldots$ \\
$\beta_{\mathrm{A} 2, \mathrm{O}}$ & $\ldots$ & $-0.34_{-0.06}^{+0.06}$ & $-1.22_{-0.18}^{+0.18}$ & $\ldots$ \\
\hline
\end{tabular}

Table 1. Extended Data: Case I (flat X-ray plateau and decaying optical plateau): Column $1-4$ : GRB names in this case. Row 1 and 2: temporal breaks in X-ray light curves at the end of the plateau phase and later time respectively. Row 3-8: temporal and spectral slopes in X-ray light curves. Row 9 and 10: temporal breaks in optical light curves at the end of the plateau phase and later time respectively. Row 9 - 14: temporal and spectral slopes in optical light curves. Errors are in one $\sigma$ significance. Optical spectral slopes are obtained from the literature $(050319,060605,060614,060729,061121$, 080310, 080607, 100418A in Ref. ${ }^{89}$; 060714 in Ref. ${ }^{96}$; 091029 in Ref. ${ }^{91}$; 110213A in Ref. ${ }^{92}$; 171205A Ref. ${ }^{94}$ ) Note: Optical temporal slopes of GRBs 080607, 091029, 110213A, 130831A are converted from $\mathrm{AB}$ magnitude to $v F_{v}$ flux by multiplying $1 / 2.5$. From the X-ray LC of 091029 , a faint flare during the plateau phase is excluded, see details in Method 'X-ray data'. The lack of the data in the X-ray LC of 130831A affects the definition of $\mathrm{T}_{\mathrm{a}, \mathrm{X}}$, but with two breaks, we could define it better $\left(\log \mathrm{T}_{\mathrm{a}, \mathrm{X}}=3.08_{-0.18}^{+0.28}\right)$ at a later time with large error bars. However, due to the poor fitting result, we used the value presented in this table. GRB 080607 has a flat slope with larger errors due to lack of data at the end of the X-ray plateau phase, so the last slope is used as the slope of self-similar phase. In addition, the first break is defined to be the $T_{a, X}$ but this break can be at later time with steeper slope during the plateau phase (see Method 'X-ray data' for further explanations). 


\begin{tabular}{cccccc}
\hline GRB name & 060614 & 060729 & 080310 & $100418 \mathrm{~A}$ & $171205 \mathrm{~A}$ \\
\hline $\log \mathrm{T}_{\mathrm{a}, \mathrm{X}}(\mathrm{s})$ & $4.53_{-0.03}^{+0.03}$ & $4.58_{-0.02}^{+0.04}$ & $4.04_{-0.04}^{+0.04}$ & $4.90_{-0.07}^{+0.11}$ & $4.96_{-0.04}^{+0.04}$ \\
$\log \mathrm{T}_{\mathrm{b}, \mathrm{X}}(\mathrm{s})$ & $5.13_{-0.09}^{+0.09}$ & $5.15_{-0.04}^{+0.04}$ & $\ldots$ & $\ldots$ & $\ldots$ \\
$\alpha_{\mathrm{p}, \mathrm{X}}$ & $0.02_{-0.05}^{+0.05}$ & $-0.14_{-0.02}^{+0.02}$ & $-0.21_{-0.10}^{+0.10}$ & $-0.09_{-0.06}^{+0.06}$ & $0.21_{-0.09}^{+0.09}$ \\
$\alpha_{\mathrm{A} 1, \mathrm{X}}$ & $-1.41_{-0.11}^{+0.13}$ & $-0.87_{-0.06}^{+0.05}$ & $-1.55_{-0.06}^{+0.06}$ & $-1.39_{-0.13}^{+0.09}$ & $-1.06_{-0.05}^{+0.05}$ \\
$\alpha_{\mathrm{A} 2, \mathrm{X}}$ & $-2.23_{-0.15}^{+0.12}$ & $-1.41_{-0.02}^{+0.02}$ & $\ldots$ & $\ldots$ & \\
$\beta_{\mathrm{p}, \mathrm{X}}$ & $0.74_{-0.05}^{+0.11}$ & $0.97_{-0.04}^{+0.04}$ & $1.14_{-0.07}^{+0.12}$ & $0.9_{-0.3}^{+0.4}$ & $0.55_{-0.10}^{+0.21}$ \\
$\beta_{\mathrm{A} 1, \mathrm{X}}$ & $0.92_{-0.15}^{+0.16}$ & $0.98_{-0.04}^{+0.04}$ & $0.89_{-0.15}^{+0.16}$ & $0.84_{-0.29}^{+0.32}$ & $0.94_{-0.22}^{+0.23}$ \\
$\log \mathrm{T}_{\mathrm{a}, \mathrm{O}}(\mathrm{s})$ & $4.47_{-0.04}^{+0.02}$ & $4.71_{-0.01}^{+0.01}$ & $3.54_{-0.10}^{+0.12}$ & $4.67_{-0.13}^{+0.10}$ & $4.55_{-0.03}^{+0.04}$ \\
$\log \mathrm{T}_{\mathrm{b}, \mathrm{O}}(\mathrm{s})$ & $4.84_{-0.03}^{+0.05}$ & $\ldots$ & $4.60_{-0.62}^{+0.27}$ & $\ldots$ & $5.25_{-0.03}^{+0.04}$ \\
$\alpha_{\mathrm{p}, \mathrm{O}}$ & $0.10_{-0.03}^{+0.04}$ & $-0.03_{-0.01}^{+0.01}$ & $-0.02_{-0.04}^{+0.04}$ & $0.22_{-0.08}^{+0.07}$ & $0.15_{-0.01}^{+0.01}$ \\
$\alpha_{\mathrm{A} 1, \mathrm{O}}$ & $-0.96_{-0.14}^{+0.16}$ & $-1.39_{-0.02}^{+0.02}$ & $-1.07_{-0.12}^{+0.05}$ & $-0.70_{-0.05}^{+0.05}$ & $-0.65_{-0.01}^{+0.01}$ \\
$\alpha_{\mathrm{A} 2, \mathrm{O}}$ & $-2.02_{-0.08}^{+0.08}$ & $\ldots$ & $-1.21_{-0.38}^{+0.23}$ & $\ldots$ & $0.44_{-0.01}^{+0.01}$ \\
$\beta_{\mathrm{p} 1, \mathrm{O}}$ & $-0.30_{0.0}^{0.0}$ & $-0.59_{0.0}^{0.0}$ & $-0.97_{-0.04}^{+0.04}$ & $\ldots$ & $-0.65_{-0.39}^{+0.39}$ \\
$\beta_{\mathrm{p} 2, \mathrm{O}}$ & $\ldots$ & $-0.88_{0.01}^{0.0}$ & $\ldots$ & $\ldots$ & $-0.83_{-0.19}^{+0.19}$ \\
$\beta_{A 1, O}$ & $-0.35_{0.0}^{0.0}$ & $-0.54_{0.0}^{0.0}$ & $-0.88_{-0.04}^{+0.04}$ & $-1.19_{-0.02}^{+0.02}$ & $-0.77_{-0.15}^{+0.15}$ \\
$\beta_{\mathrm{A} 2, \mathrm{O}}$ & $\ldots$ & $\ldots$ & $\ldots$ & $\ldots$ & $-0.88_{-0.60}^{+0.60}$ \\
\hline
\end{tabular}

Table 2. Extended Data: Case II (both X-ray and optical plateaus are flat): Columns and Rows are as defined in Table 1. Optical temporal slopes for GRBs 100418A and 171205A are converted from AB magnitude to $v F_{v}$ flux by multiplying by $1 / 2.5$. For GRB $171205 \mathrm{~A}$, we point out that the rise of the supernovae bump in optical band after 2 days ${ }^{94}$ affects the slopes in both phases during the plateau (slightly rising) and self-similar phases (decaying more slowly than -1.2) as well as the definition of $T_{a}$ which is quite early in the optical band than in the X-ray band. 


\begin{tabular}{ccccc}
\hline & 050319 & 060605 & 060714 & 061121 \\
\hline $\log \mathrm{T}_{\mathrm{a}, \mathrm{X}}(\mathrm{s})$ & $4.50_{-0.06}^{+0.06}$ & $3.69_{-0.11}^{+0.16}$ & $3.65_{-0.10}^{+0.14}$ & $3.96_{-0.03}^{+0.03}$ \\
$\log \mathrm{T}_{\mathrm{b}, \mathrm{X}}(\mathrm{s})$ & $\ldots$ & $4.18_{-0.06}^{+0.09}$ & $\ldots$ & $\ldots$ \\
$\alpha_{\mathrm{p}, \mathrm{X}}$ & $-0.55_{-0.02}^{+0.02}$ & $-0.41_{-0.04}^{+0.08}$ & $-0.42_{-0.11}^{+0.10}$ & $-0.59_{-0.01}^{+0.02}$ \\
$\alpha_{\mathrm{A} 1, \mathrm{X}}$ & $-1.44_{-0.12}^{+0.11}$ & $-1.24_{-0.25}^{+0.15}$ & $-1.24_{-0.04}^{+0.03}$ & $-1.42_{-0.02}^{+0.02}$ \\
$\alpha_{\mathrm{A} 2, \mathrm{X}}$ & $\ldots$ & $-2.25_{-0.17}^{+0.14}$ & $\ldots$ & $\ldots$ \\
$\beta_{\mathrm{p}, \mathrm{X}}$ & $1.00_{-0.06}^{+0.09}$ & $0.90_{-0.07}^{+0.14}$ & $0.88_{-0.14}^{+0.15}$ & $0.84_{-0.28}^{+0.30}$ \\
$\beta_{\mathrm{A} 1, \mathrm{X}}$ & $1.18_{-0.20}^{+0.21}$ & $1.16_{-0.17}^{+0.17}$ & $1.08_{-0.19}^{+0.20}$ & $0.85_{-0.07}^{+0.07}$ \\
$\beta_{\mathrm{A} 2, \mathrm{X}}$ & $\ldots$ & $1.08_{-0.15}^{+0.20}$ & $\ldots$ & $0.77_{-0.10}^{+0.10}$ \\
$\log \mathrm{T}_{\mathrm{a}, \mathrm{O}}(\mathrm{s})$ & $4.55_{-0.04}^{+0.04}$ & $3.47_{-0.02}^{+0.05}$ & $3.77_{-0.18}^{+0.16}$ & $4.14_{-0.32}^{+0.35}$ \\
$\alpha_{\mathrm{p}, \mathrm{O}}$ & $-0.59_{-0.01}^{+0.01}$ & $-1.02_{-0.02}^{+0.01}$ & $-0.27_{-0.09}^{+0.09}$ & $-0.48_{-0.05}^{+0.05}$ \\
$\alpha_{\mathrm{A} 1, \mathrm{O}}$ & $-0.64_{-0.014}^{+0.017}$ & $-1.61_{-0.02}^{+0.01}$ & $-0.62_{-0.28}^{+0.27}$ & $-1.08_{-0.64}^{+0.27}$ \\
$\beta_{\mathrm{p}, \mathrm{O}}$ & $-0.36_{0.0}^{0.0}$ & $-1.32_{-0.03}^{+0.03}$ & $-0.44_{-0.04}^{+0.04}$ & $-0.68_{-0.06}^{+0.06}$ \\
$\beta_{\mathrm{A} 1, \mathrm{O}}$ & $-0.61_{0.0}^{0.0}$ & $-1.14_{-0.02}^{+0.02}$ & $-0.98_{-0.11}^{+0.11}$ & $-0.68_{-0.02}^{+0.02}$ \\
\hline
\end{tabular}

Table 3. Extended Data: Case III (both X-ray and optical plateaus are decaying): Columns and Rows are as defined in Table 1. Note: Optical temporal slopes of GRBs 050319 and GRB 060605 are converted from $\mathrm{AB}$ magnitude to $v F_{v}$ flux by multiplying $1 / 2.5$. The window timing (WT) data from the LC of 061121 are excluded during the fit, see details in Method 'X-ray data'. GRB 060605 has a flare at the beginning of the plateau phase between 350 and 600s (see Method 'X-ray data' for more comments). This time corresponds to the bump in the optical band with end break time at around $586_{-43}^{+31} \mathrm{~s}$ and slope $\left(0.18_{-0.02}^{+0.01}\right)$. We therefore consider the later break to be $\mathrm{T}_{\mathrm{a}, \mathrm{O}}$ and the later slope is the slope of the plateau phase in optical band before the last break and last slope. However, it is important to note that this bump effects the later slopes during both the plateau and self-similar phases.

\begin{tabular}{|c|c|c|}
\hline Scenario & Coasting & decay \\
\hline [A] $\quad v<v_{c}<v_{m}$ & $F_{V} \propto t_{\text {obs. }}^{-1 / 3} v^{1 / 3}$ & $F_{v} \propto t_{\mathrm{obs}}^{-2 / 3} v^{1 / 3}$ \\
\hline [B] $v_{c}<v<v_{m}$ & $F_{V} \propto t_{\text {obs }}^{1 / 2} v^{-1 / 2}$ & $F_{V} \propto t_{\text {obs. }}^{-1 / 4} v^{-1 / 2}$ \\
\hline [C] $\quad v_{c}<v_{m}<v$ & $F_{v} \propto t_{\text {obs. }}^{(2-p) / 2} v^{-p / 2} \sim t_{\text {obs. }}^{0 . .-0.2}$ & $F_{V} \propto t_{\text {obs. }}^{(2-3 p) / 4} v^{-p / 2} \sim t_{\text {obs. }}^{-1 . .-1.3}$ \\
\hline [D] $v<v_{m}<v_{c}$ & $F_{v} \propto t_{\text {obs. }}^{1 / 3} v^{1 / 3}$ & $F_{V} \propto t_{\text {obs. }}^{0} v^{1 / 3}$ \\
\hline$v_{m}<v<v_{c}$ & $F_{V} \propto t_{\text {obs. }}^{(1-p) / 2} v^{-(p-1) / 2} \sim t_{\text {obs. }}^{-0.5 . .-0.7}$ & $F_{v} \propto t_{\text {obs. }}^{(1-3 p) / 4} v^{-(p-1) / 2} \sim t_{\text {obs. }}^{-1.25 . .-1.55}$ \\
\hline$[\mathrm{F}] \quad v_{m}<v_{c}<v$ & $F_{V} \propto t_{\mathrm{obs} .}^{(2-p) / 2} v^{-p / 2} \sim t_{\mathrm{obs}}^{0 . .-0.2}$ & $F_{v} \propto t_{\mathrm{obs} .}^{(2-3 p) / 4} v^{-p / 2} \sim t_{\mathrm{obs}}^{-1 . .-1.3}$ \\
\hline
\end{tabular}

Table 4. Extended Data: Temporal and spectral evolution in each of the possible six spectral regimes (regions). Here, $p$ is the power law index of the accelerated electrons. Expected values based on both theory ${ }^{110}$ and observations ${ }^{111}$ suggest $2.0 \leq p \lesssim 2.4$. We indicate the expected temporal evolution in regions $[\mathrm{C}],[\mathrm{E}]$ and $[\mathrm{F}]$ for power law index in this range. 


\begin{tabular}{|c|c|c|c|c|c|}
\hline GRB name & region in X-ray band & $p_{\mathrm{X}}$ & region in optical band & $p_{\mathrm{U}}$ & note \\
\hline \multicolumn{6}{|c|}{ Listed in case I } \\
\hline 080607 & $\mathrm{~F}$ & $\sim 2.5$ & $\mathrm{E}$ & 2.0 & \\
\hline 091029 & $\mathrm{~F}$ & 2.3 & $\mathrm{~F}$ & $2.4($ E 1.8$)$ & may transit to $\mathrm{E}$ \\
\hline 110213 & $\mathrm{~F}$ & $\sim 2.0$ & $\mathrm{E}$ & 2.1 & \\
\hline 130831 & $\mathrm{~F}$ & 2.0 & $\mathrm{E}$ & 2.1 & \\
\hline \multicolumn{6}{|c|}{ Listed in case II } \\
\hline 060614 & $\mathrm{~F}$ & $\sim 2.3$ & $\mathrm{~F}$ & 2.0 & \\
\hline 060729 & $\mathrm{~F}$ & 2.0 & $\mathrm{~F}$ & 2.3 & \\
\hline 080310 & $\mathrm{~F}$ & $\sim 2.5$ & $\mathrm{~F}$ & 2.0 & \\
\hline 100418 & $\mathrm{~F}$ & 2.2 & $\mathrm{~F}$ & $\sim 1.8$ & \\
\hline 171205 & $\mathrm{~F}$ & 2.0 & $\mathrm{~F}$ & $\sim 1.8$ & \\
\hline \multicolumn{6}{|c|}{ Listed in case III } \\
\hline 050319 & $\mathrm{E}$ & 2.1 & $\mathrm{E}$ & $\sim 1.8-2.1$ & \\
\hline 060605 & $\mathrm{E}$ & 2.0 & $\mathrm{E}$ & $\sim 2.1$ & \\
\hline 060714 & $\mathrm{E}$ & 2.0 & $\mathrm{E}$ & $\sim 2.0$ & \\
\hline 061121 & $\mathrm{E}$ & $\sim 2.1$ & $\mathrm{E}$ & 2.0 & \\
\hline
\end{tabular}

Table 5. Region and electron power law index $(p)$ in both X-ray and optical bands. In Column 1, GRB names are ordered depending on the cases (I, II, III listed in Tables 1, 2, 3 respectively). From Column 2 to 5, regions and electron power law indices $(p)$ are defined for the X-ray and optical data respectively.

\begin{tabular}{|c|c|c|c|c|c|c|c|c|c|c|c|}
\hline GRB name & $\bar{z}$ & $\begin{array}{c}\mathrm{d}_{\mathrm{L}} \\
(\mathrm{Mpc})\end{array}$ & $\begin{array}{c}\mathrm{S}(15-150 \mathrm{keV}) \\
\left(10^{-7} \mathrm{erg} \mathrm{cm}^{-2}\right)\end{array}$ & $\alpha_{\gamma}$ & $\begin{array}{c}\log \left(\mathrm{E}_{\text {iso }}\right) \\
\text { (erg) }\end{array}$ & $\begin{array}{c}T_{a_{3} X} \\
\left(10^{3} s\right)\end{array}$ & $\begin{array}{c}v F_{V}(\mathrm{X}) \\
\left(10^{-12} \mathrm{erg} \mathrm{cm}^{-2} \mathrm{~s}^{-1}\right)\end{array}$ & $\begin{array}{c}v F_{v}(\mathrm{U}) \\
\left(10^{-13} \mathrm{erg} \mathrm{cm}^{-2} \mathrm{~s}^{-1}\right)\end{array}$ & $\begin{array}{c}\mathrm{T}_{\text {ref.,U }} \\
(\mathrm{s})\end{array}$ & $\begin{array}{c}v F_{v}(\mathrm{X}) \\
\left(10^{-12} \mathrm{erg} \mathrm{cm}^{-2} \mathrm{~s}^{-1}\right)\end{array}$ & $\begin{array}{c}v F_{V}(\mathrm{U}) \\
\left(10^{-12} \mathrm{erg} \mathrm{cm}^{-2}\right.\end{array}$ \\
\hline \multicolumn{12}{|c|}{ Listed in case I } \\
\hline 080607 & 3.036 & 26150 & $240 \pm 0.0$ & $1.31 \pm 0.04$ & $53.27 \pm 0.02$ & $1.56_{-0.15}^{+0.20}$ & $6.81 \pm 1.53$ & $0.39 \pm 0.18$ & 1010 & $82.7 \pm 18.5$ & $0.18 \pm 0.01$ \\
\hline 091029 & 2.752 & 23222 & $24 \pm 1.0$ & $1.46 \pm 0.27$ & $52.31 \pm 0.16$ & $14.2_{-3.45}^{+1.50}$ & $1.34 \pm 0.23$ & $1.17 \pm 0.29$ & 1170 & $2.8 \pm 0.6$ & $0.3 \pm 0.1$ \\
\hline $110213 \mathrm{~A}$ & 1.46 & 10662 & $59 \pm 4.0$ & $1.83 \pm 0.12$ & $52.45 \pm 0.06$ & $1.35_{-0.18}^{+0.14}$ & $350 \pm 77$ & $8.45 \pm 0.33$ & 1130 & $218 \pm 48$ & $7.7 \pm 0.4$ \\
\hline $130831 \mathrm{~A}$ & 0.4791 & 2704 & $65 \pm 0.2$ & $1.93 \pm 0.05$ & $51.57 \pm 0.01$ & $0.75_{-0.07}^{+0.08}$ & $259 \pm 56$ & $462 \pm 28$ & 732 & $259 \pm 56$ & $46.2 \pm 2.8$ \\
\hline \multicolumn{12}{|c|}{ Listed in case II } \\
\hline 060614 & 0.125 & 586 & $204 \pm 3.6$ & $2.02 \pm 0.04$ & $50.87 \pm 0.01$ & $34.1_{-2.63}^{+2.27}$ & $3.28 \pm 0.63$ & $6.26 \pm 1.43$ & 4838 & $2.1 \pm 0.5$ & $49.9 \pm 11.6$ \\
\hline 060729 & 0.54 & 3124 & $26 \pm 2.1$ & $1.75 \pm 0.14$ & $51.25 \pm 0.04$ & $38.0_{-2.08}^{+3.18}$ & $6.35 \pm 1.43$ & $33.4 \pm 7.5$ & 1160 & $10.6 \pm 2.4$ & $4.13 \pm 0.94$ \\
\hline 080310 & 2.42 & 19862 & $23 \pm 2.0$ & $2.32 \pm 0.16$ & $52.67 \pm 0.09$ & $10.9_{-0.88}^{+0.94}$ & $3.41 \pm 0.76$ & $2.58 \pm 0.59$ & 1505 & $4.9 \pm 0.9$ & $14.5 \pm 3.2$ \\
\hline $100418 \mathrm{~A}$ & 0.62 & 3695 & $3.4 \pm 0.5$ & $2.16 \pm 0.25$ & $50.57 \pm 0.08$ & $79.3_{-12.8}^{+20.6}$ & $0.86 \pm 0.22$ & $2.58 \pm 0.05$ & 1000 & $0.15 \pm 0.04$ & $0.06 \pm 0.01$ \\
\hline $171205 \mathrm{~A}$ & 0.0368 & 162 & $36 \pm 3.0$ & $1.41 \pm 0.14$ & $49.03 \pm 0.04$ & $91.0_{-8.45}^{+8.60}$ & $1.02 \pm 0.24$ & $5.68 \pm 0.68$ & 10834 & $0.61 \pm 0.16$ & $1.44 \pm 0.09$ \\
\hline \multicolumn{12}{|c|}{ Listed in case III } \\
\hline 050319 & 3.24 & 28280 & $13.1 \pm 1.5$ & $2.02 \pm 0.19$ & $52.48 \pm 0.13$ & $32.0_{-4.25}^{+4.17}$ & $1.24 \pm 0.28$ & $1.44 \pm 0.26$ & 1120 & $5.02 \pm 1.13$ & $1.20 \pm 0.17$ \\
\hline 060605 & 3.78 & 34014 & $7.0 \pm 0.9$ & $1.55 \pm 0.20$ & $52.00 \pm 0.15$ & $4.88_{-1.85}^{+1.24}$ & $5.25 \pm 1.19$ & $9.51 \pm 1.14$ & 534 & $16.3 \pm 3.3$ & $9.51 \pm 0.42$ \\
\hline 060714 & 2.710 & 22793 & $28.3 \pm 1.7$ & $1.93 \pm 0.11$ & $52.64 \pm 0.10$ & $4.50_{-0.99}^{+1.37}$ & $13.3 \pm 2.92$ & $1.80 \pm 0.53$ & 1069 & $13.6 \pm 3.07$ & $0.35 \pm 0.08$ \\
\hline 061121 & 1.314 & 9355 & $137 \pm 2.0$ & $1.41 \pm 0.03$ & $52.58 \pm 0.01$ & $9.11_{-0.64}^{+0.53}$ & $61.5 \pm 12.3$ & $9.16 \pm 2.50$ & 1173 & $67 \pm 15$ & $1.66 \pm 0.40$ \\
\hline
\end{tabular}

Table 6. Extended Data: Some key parameters of the 13 GRBs in our sample. Columns $1-12$ are the GRB name, redshift, luminosity distance, fluence, photon spectral index, isotropic equivalent energy, time at the end of the X-ray plateau phase, $v F_{v}$ X-ray flux and $v F_{v}$ optical flux at $\mathrm{T}_{\mathrm{a}, \mathrm{X}}$, a reference time in optical band at around $1000 \mathrm{~s}, v F_{v} \mathrm{X}$-ray flux and $v F_{v}$ optical flux at $\mathrm{T}_{\text {ref.,U }}$ respectively. Note that the fluence of GRB 080607 has no error in the online Swift GRB table. See Method 'Flux Ratio' for the definition of each parameters. 


\begin{tabular}{|c|c|c|c|c|c|c|c|c|c|}
\hline & & $\varepsilon_{B}=1.0$ & & $\varepsilon_{B}=0.1$ & & & $\varepsilon_{B}=0.01$ & & \\
\hline GRB name & $\varepsilon_{e}$ & $\Gamma_{i}$ & $\overline{A_{\star}}$ & $\Gamma_{i}$ & $\overline{A_{\star}}$ & $\varepsilon_{e}$ & $\Gamma_{i}$ & $\overline{A_{\star}}$ & $\begin{array}{c}\text { Flux ratio } \\
v F_{V}(\mathrm{X}) / v F_{v}(\mathrm{U}) \\
\end{array}$ \\
\hline Listed in case I & direct value & direct value & direct value & direct value & direct value & & direct value & direct value & \\
\hline 080607 & $1.3 \times 10^{-2}$ & 503 & $5 \times 10^{-5}$ & 322 & $3 \times 10^{-4}$ & & 238 & $1 \times 10^{-3}$ & $174 \pm 89$ \\
\hline 091029 & $3.1 \times 10^{-2}$ & 137 & $1 \times 10^{-4}$ & 77 & $1 \times 10^{-3}$ & & 52 & $5 \times 10^{-3}$ & $11.5 \pm 3.5$ \\
\hline $110213 \mathrm{~A}$ & $5.1 \times 10^{-2}$ & 361 & $2 \times 10^{-5}$ & 203 & $2 \times 10^{-4}$ & & 136 & $1 \times 10^{-3}$ & $41.4 \pm 0.2$ \\
\hline $130831 \mathrm{~A}$ & $2.7 \times 10^{-2}$ & 113 & $3 . \times 10^{-4}$ & 84 & $1 \times 10^{-3}$ & & 56 & $5 \times 10^{-3}$ & $5.6 \pm 1.3$ \\
\hline Listed in case II & direct value & upper limit & lower limit & & & & upper limit & lower limit & \\
\hline 060614 & $3 \times 10^{-3}$ & 15 & $3 \times 10^{-3}$ & & & & 8 & $5 \times 10^{-2}$ & $5.2 \pm 1.6$ \\
\hline 060729 & $1.5 \times 10^{-1}$ & 20 & $3 \times 10^{-3}$ & & & & 10 & $5 \times 10^{-2}$ & $1.9 \pm 0.6$ \\
\hline 080310 & $1.5 \times 10^{-2}$ & 82 & $2 \times 10^{-3}$ & & & & 37 & $5 \times 10^{-2}$ & $13.2 \pm 4.2$ \\
\hline 100418A & $2.7 \times 10^{-2}$ & 13 & $2 \times 10^{-3}$ & & & & 6 & $5 \times 10^{-2}$ & $3.35 \pm 0.86$ \\
\hline \multirow[t]{2}{*}{$171205 \mathrm{~A}$} & $1.4 \times 10^{-2}$ & 5 & $1 \times 10^{-3}$ & & & & 2 & $5 \times 10^{-2}$ & $1.8 \pm 0.5$ \\
\hline & $\varepsilon_{B}=0.001$ & $\varepsilon_{B}=0.001$ & & & & $\varepsilon_{B}=0.01$ & $\varepsilon_{B}=0.01$ & & \\
\hline Listed in case III & lower limit & lower limit & upper limit & & & lower limit & lower limit & upper limit & $86+25$ \\
\hline $\begin{array}{l}050319 \\
060605\end{array}$ & $\begin{array}{l}1.6 \times 10^{-2} \\
2.6 \times 10^{-2}\end{array}$ & $\begin{array}{l}60 \\
66\end{array}$ & $3 \times 10^{-3}$ & & & $\begin{array}{l}3.1 \\
3.8\end{array}$ & $\begin{array}{c}91 \\
104\end{array}$ & $\begin{array}{l}3 \times 10^{-4} \\
5 \times 10^{-4}\end{array}$ & $\begin{array}{l}0.0 \pm 2.5 \\
5.5 \pm 1.4\end{array}$ \\
\hline 060714 & $5.5 \times 10^{-4}$ & 94 & $3 \times 10^{-3}$ & & & $8 \times 10^{-2}$ & 148 & $5 \times 10^{-4}$ & $74 \pm 27$ \\
\hline 061121 & $1.9 \times 10^{-3}$ & 68 & $3 \times 10^{-3}$ & & & $2.8 \times 10^{-1}$ & 106 & $5 \times 10^{-4}$ & $67 \pm 23$ \\
\hline
\end{tabular}

Table 7. Extended Data: Deduced parameters of the outflow and wind. $\varepsilon_{e}$ is the fraction of energy in the electrons, $A_{\star}$ is the wind density, $\Gamma_{i}$ is the initial jet Lorentz factor. Direct value of $\varepsilon_{e}$ is computed by using the information in the optical data for the GRBs listed in case I and II respectively. In addition, direct values of $A_{\star}$ and $\Gamma_{i}$ are obtained by assuming the fraction of energy in the magnetic field is $\varepsilon_{B}=1.0,0.1,0.01$ for the GRBs listed in case I. Moreover, an (external) upper limit on $\varepsilon_{B}$, e.g., $\varepsilon_{B} \leq 1.0$ is used to compute upper limit on $\Gamma_{i}$ and lower limit on $A_{\star}$ for the GRBs listed in case II. Vice-versa, external knowledge on lower limits (e.g., $\varepsilon_{B} \geq 0.01$ and $\varepsilon_{B} \geq 0.001$ ) can be used to compute lower limit on the value of $\Gamma_{i}$ and an upper limit on $A_{\star}$ as well as lower limit on $\varepsilon_{e}$ for the GRBs listed in case III (see Method 'Theoretical model'). Flux ratio is the ratio of the X-ray and optical $\left(v F_{v}\right)$ fluxes calculated at $T_{a, X}$ (see Table 6). They are consistent with the theoretical predictions in all three different cases.

\begin{tabular}{ccccccc}
\hline $\log \left(\mathrm{T}_{\mathrm{a}}\right)$ & $\log \left(\mathrm{T}_{\mathrm{b}}\right)$ & $\mathrm{N}$ & $\alpha_{\mathrm{p}}$ & $\alpha_{\mathrm{A} 1}$ & $\alpha_{\mathrm{A} 2}$ & $\log (\mathrm{V})$ \\
\hline $4.53_{-0.03}^{+0.03}$ & $5.13_{-0.09}^{+0.09}$ & $-0.68_{-0.02}^{+0.02}$ & $0.02_{-0.05}^{+0.05}$ & $-1.41_{-0.11}^{+0.13}$ & $-2.23_{-0.15}^{+0.12}$ & $-11.54_{-2.55}^{+2.34}$ \\
\hline
\end{tabular}

Table 8. Extended Data: X-ray temporal fit parameters of GRB 060614 with the Bayesian analysis tool emcee. $\log \left(\mathrm{T}_{\mathrm{a}}\right)$ is the break time at the end of the plateau phase and $\log \left(\mathrm{T}_{\mathrm{b}}\right)$ is the jet break time. $\mathrm{N}$ is the normalization of the fit. The slopes $\alpha_{\mathrm{p}}, \alpha_{\mathrm{A} 1}, \alpha_{\mathrm{A} 2}$ are obtained during the plateau phase, self-similar phase and after the jet break time respectively. $\mathrm{V}$ is the intrinsic scatter of the data. 


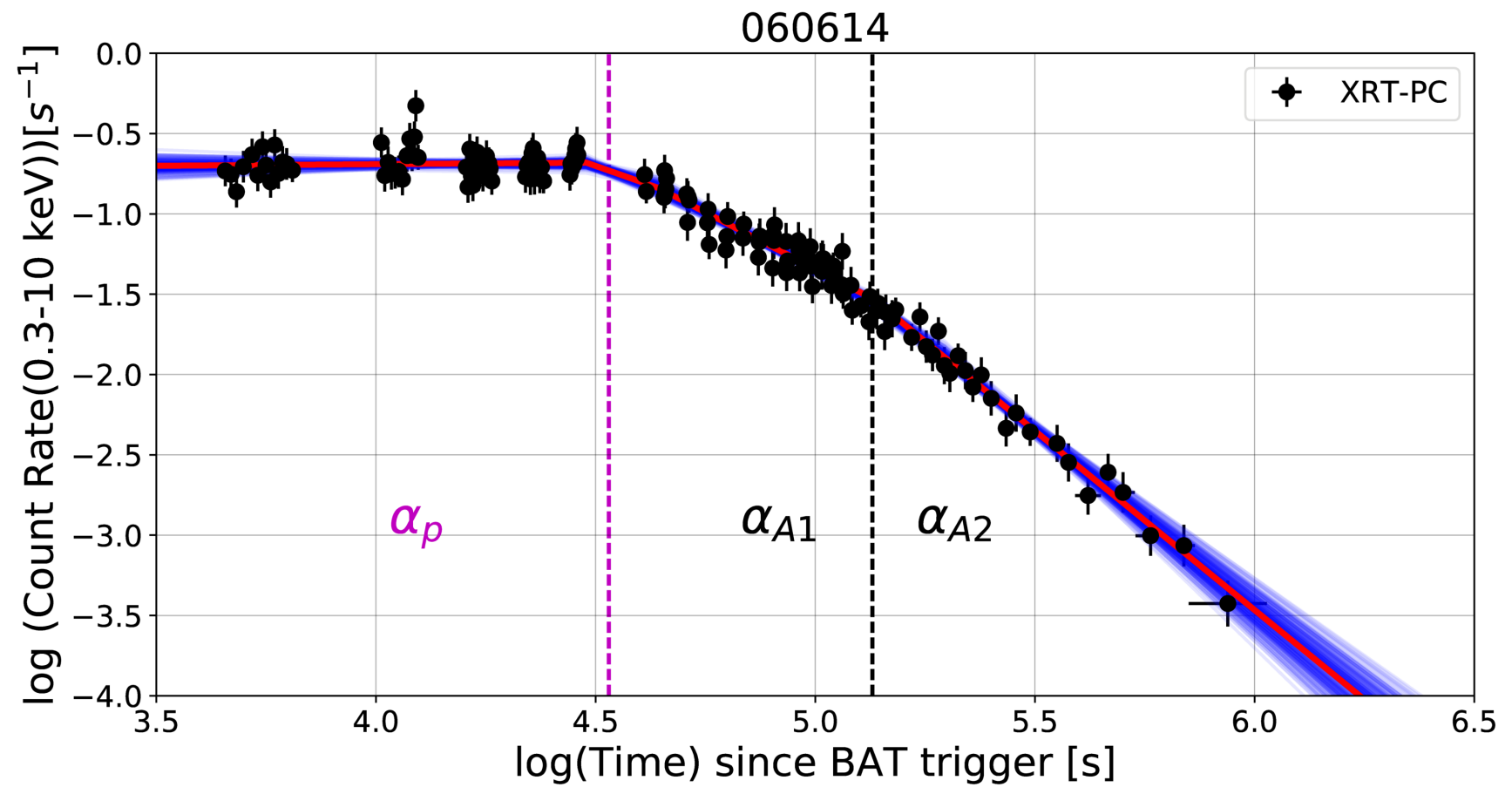

Figure 9. Extended Data: An example: The LC of GRB 060614 fitted with a Bayesian analysis tool emcee. The black points represent the data (count-rate vs time), the red line shows the mean of the posterior distribution, blue lines are 3200 randomly selected samples from the MCMC sampling. The dashed lines (purple and black) represent the break times $\log \left(\mathrm{T}_{\mathrm{a}}\right.$ ) (at the end of the plateau phase) and $\log \left(\mathrm{T}_{\mathrm{b}}\right)$ respectively. The $\alpha_{\mathrm{p}}$ (purple), $\alpha_{\mathrm{A} 1}$ (black), $\alpha_{\mathrm{A} 2}$ (black) are slopes during the plateau phase, self-similar phase and after the jet break time respectively.

\begin{tabular}{cccc}
\hline GRB name & $A_{\mathrm{U}, \text { Gal. }}$ & $A_{\mathrm{U}, \text { host }}$ & note \\
\hline 050319 & 0.052 & $0.53 \pm 0.012$ & (V-band) $^{89}$ \\
060605 & 0.255 & $1.33 \pm 0.10$ & (CR-band $^{89}$ \\
060614 & 0.107 & $0.23 \pm 0.18$ & (U-band) $^{89}$ \\
060714 & 0.378 & $1.53_{-0.58}^{+0.65}$ & $A v_{\text {host }}=0.79_{-0.35}^{+0.39}$ is given in Ref. \\
08 & (U-band) $^{89}$ \\
060729 & 0.272 & $0.433 \pm 0.18$ & (U-band) $^{89}$ \\
061121 & 0.226 & $0.92 \pm 0.30$ & (U-band) $^{89}$ \\
080310 & 0.196 & $0.433 \pm 0.18$ & (Rc-band) $^{89}$ \\
080607 & 0.111 & $2.98 \pm 0.37$ & (white-band) $^{89}$ \\
091029 & 0.08 & $\ldots$ & (U-band) no host galaxy extion $^{91}$ \\
$100418 \mathrm{~A}$ & 0.362 & $1.2 \pm 0.43$ & (U-band) no host galaxy extinction $^{92}$ \\
$110213 \mathrm{~A}$ & 1.612 & $\ldots$ & (U-band) $^{94}$ \\
$130831 \mathrm{~A}$ & 0.223 & $0.103 \pm 0.0513$ & (U-band) low host galaxy extinction $^{93}$ \\
$171205 \mathrm{~A}$ & 0.251 & 0.103 & \\
\hline
\end{tabular}

Table 9. Extended Data: Galactic and host galaxy extinctions of 13 GRBs in our sample. Column 1: GRB name, Columns 2 and 3: Galactic and host galaxy extinctions in U band. Column 4: band passes used in our analyses and references. 


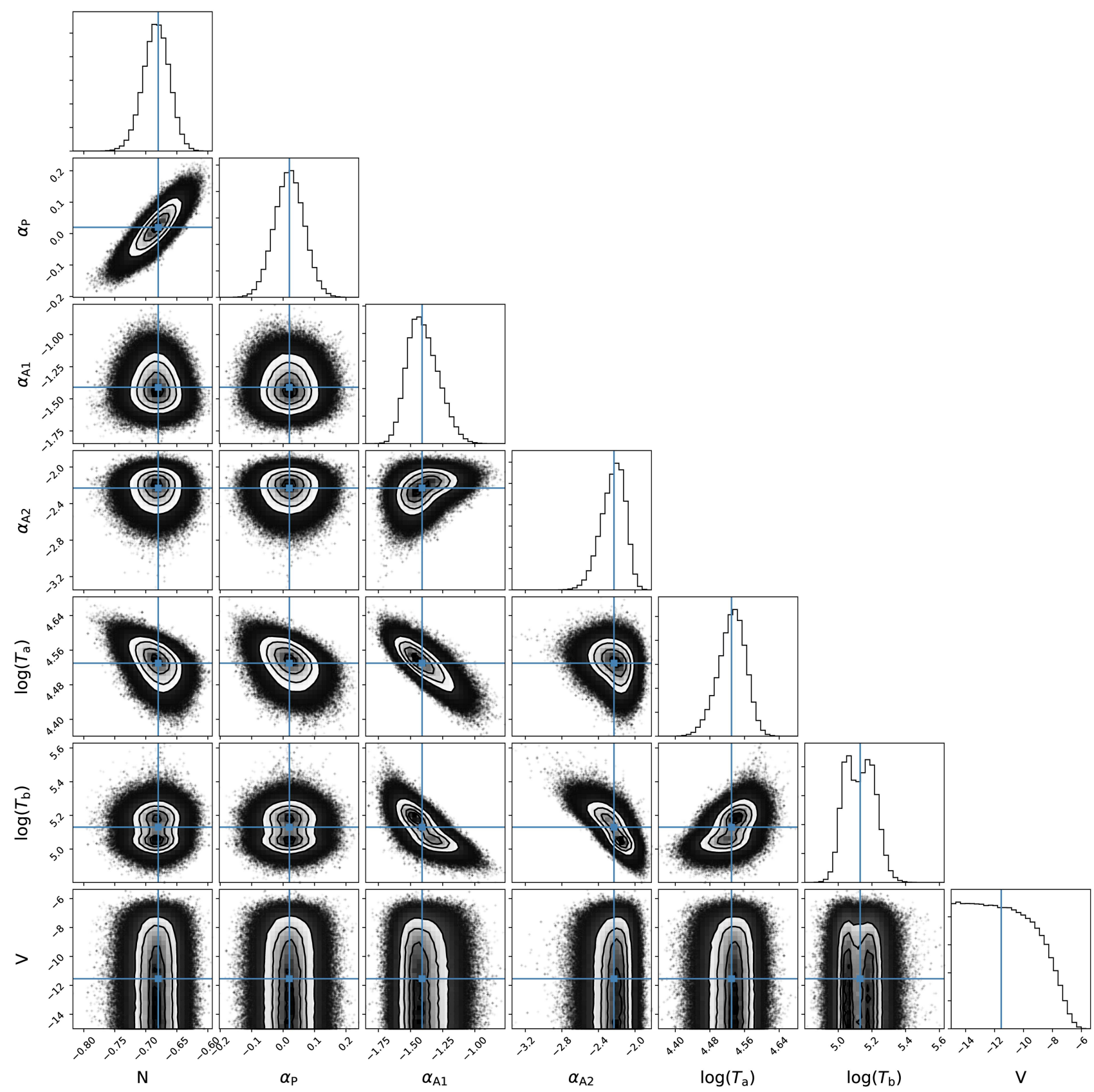

Figure 10. Extended Data: Standard emcee corner plot showing the one-and two-dimensional posterior probability distributions for the fit parameters obtained from the LC of GRB 060614. The blue lines present the mean value of each parameter from Table 8 . The density of the points and contours correlate with the posterior probability distribution from a 3200 step run of the emcee sampler. Counters gives from inward to outward 39.3\%, 68.3\%,90.3\% of the volume of the Gaussian, given the 2 dimensional nature of the data. 